\title{
The Effect of Biostimulants on the Health Status and Content of Chlorogenic Acids in Potato Tubers (Solanum Tuberosum L.) with Colored Flesh
}

\author{
Małgorzata Głosek-Sobieraj ${ }^{1}$ - Bożena Cwalina-Ambroziak ${ }^{1}$ Agnieszka Waśkiewicz² Karel Hamouz $^{3}$. \\ Adam Perczak ${ }^{2}$
}

Received: 4 June 2018 / Accepted: 7 November 2018 / Published online: 16 January 2019

(C) The Author(s) 2018

\begin{abstract}
The aim of this study was to evaluate the influence of cultivar and biostimulants on the health status of potato tubers after harvest and after 5 months of storage. The fungal pathogens isolated from potato tuber were cultured on PDA. The biostimulants limited the symptoms of dry rot in cv. Satina after harvest, in stored cv. Irga (Bio-Algeen S-90) and cv. Blaue St. Galler (Kelpak SL). The symptoms of common scab were reduced in stored potatoes cv. Satina in the Asahi SL treatment and the symptoms of late blight in stored potatoes cv. Satina in the Kelpak SL and Trifender WP treatment and in cv. Valfi in the Bio-Algeen S-90 and Kelpak SL treatment. Asahi SL and Kelpak SL decreased the severity of black scurf in stored potatoes cv. Irga. Biostimulants decreased the occurrence frequency of the causative agents of dry rot and black scurf after harvest. Total chlorogenic acid, which is predominant in potato tubers, was present in higher concentrations in the skin than in the flesh. Potato tubers had the highest content of 5-caffeoylquinic acid, followed by neochlorogenic acid (3-caffeoylquinic acid) and cryptochlorogenic acid (4-caffeoylquinic acid). Higher concentrations of chlorogenic acid were found in potato cultivars with blue-purple- and red-colored flesh than in those with yellow- and cream-colored flesh, and in response to the application of the Asahi SL biostimulant and Trifender WP.
\end{abstract}

Keywords Potato tubers $\cdot$ Cultivars $\cdot$ Biostimulants $\cdot$ Diseases $\cdot$ Fungi $\cdot$ Chlorogenic acid

Małgorzata Głosek-Sobieraj

malgorzata.glosek@uwm.edu.pl

1 Department of Entomology, Phytopathology and Molecular

Diagnostics, University of Warmia and Mazury, Olsztyn,

Poland

2 Department of Chemistry, Poznań University of Life Sciences, Poznań, Poland

3 Department of Plant Production, Food and Natural Resources, Czech University of Life Sciences, Prague, Czech Republic 


\section{Die Wirkung von Biostimulatoren auf den Gesundheitsstatus von und den Gehalt an Chlorogensäure in farbigen Kartoffelknollen (Solanum Tuberosum L.)}

\section{Zusammenfassung}

Ziel dieser Studie war es, den Einfluss der Sorte und von Biostimulatoren auf den Gesundheitsstatus von Kartoffelknollen nach der Ernte und nach 5 Monaten Lagerung zu bewerten. Die aus der Kartoffelknolle isolierten Pilzerreger wurden auf PDA kultiviert. Die Biostimulatoren verringerten die Symptome von Trockenfäule auf Knollen der Sorte Satina nach der Ernte und auf den gelagerten Knollen der Sorte Irga (Bio-Algeen S-90) und der Sorte Blaue St. Galler (Kelpak SL). Auf den gelagerten Knollen der Sorte Satina wurde eine Reduktion der Symptome des Kartoffelschorfs (Asahi SL) und der Knollenfäule (Kelpak SL und Trifender WP) festgestellt. Bei der Sorte Valfi (Bio-Algeen S-90 und Kelpak SL) waren die Symptome der Knollenfäule ebenfalls verringert. Unter dem Einfluss der Präparate Asahi SL und Kelpak SL wurde die Intensität der Pockennarbigkeit auf den aufbewahrten Knollen der Sorte Irga reduziert. Die Biostimulatoren verringerten die Häufigkeit der Erreger von Trockenfäule und Pockennarbigkeit nach der Ernte. Die in Kartoffelknollen dominierende Chlorogensäure war in größerer Menge in der Schale als im Fruchtfleisch zu finden. Den größten Anteil machte 5-Chlorogensäure aus, gefolgt von Neo-Chlorogensäure (3-Chlorogensäure) und Krypto-Chlorogensäure (4-Chlorogensäure). Höhere Chlorogensäure-Konzentrationen wurden in lila-blauen und roten Kartoffelknollen im Vergleich zu gelben und cremefarbigen Kartoffelknollen gefunden, und unter dem Einfluss von Asahi SL und Trifender WP.

\section{Schlüsselwörter Kartoffelknollen · Sorten · Biostimulatoren · Krankheiten · Pilze · Chlorogensäure}

\section{Introduction}

The health status of potatoes determines tuber yields and their quality. Pectinolytic bacteria Pectobacterium carotovorum subsp. carotovorum, $P$. atrosepticum and Dickeya sp. cause soft rot which causes significant losses in stored potatoes (Sławiak et al. 2009). In a supportive environment, the above pathogens can latently infect seed potatoes and seedlings (Gardan et al. 2003). Common scab is caused by a wide variety of Streptomyces species which are ubiquitous and highly virulent pathogens in potato farms (Cullen and Lees 2007). Potato cultivars are characterized by different susceptibility/resistance to common scab. In the group of around 20 identified species of the genus Streptomyces, S. scabies, $S$. acidiscabiei and S. turgidiscabiei are the most prevalent causative agents of common scab in potatoes (Lutomirska 2008). According to Leiminger et al. (2013), the most pathogenic isolates of Streptomes in Germany include S. europaeiscabiei (predominant species), S. stelliscabiei, S. acidiscabiei, S. turgidiscabiei and S. bottropensis. Dry rot caused by several species of the genera Fusarium and Gibberella is the most serious disease in stored potatoes which decreases potato yields by $6-25 \%$ or even up to $60 \%$ during long-term storage (Gachango et al. 2012). Rhizoctonia solani sclerotia overwinter on infected potato tubers; they can survive in soil for several years and infect healthy plants (Larkin and Honeycutt 2006).

In modern potato farms, the selection of resistant cultivars and the use of biodegradable crop protection agents play equally important roles in disease prevention as agronomic practices, including crop rotation and organic and mineral fertilization. Potato cultivars with colored flesh ap- pear to be more resistant to environmental stressors, including pathogenic infections (Tierno and Ruiz de Galarreta 2016). According to Hamouz et al. (2010) and Bellumori et al. (2017), the above can be attributed to the fact that potatoes with colored flesh contain 2 to 10 times more phenolic acids than cultivars with yellow flesh. Terry et al. (2014) demonstrated that phenolic compounds increased plant resistance to pathogens. Phenolic acids are the most abundant phenolic compounds in potato tubers (Singh and Saldana 2011). Chlorogenic acid -ester of caffeic acid and quinic acid, is the predominant phenolic acid identified in potato tubers (Amado et al. 2014). Recent years have witnessed an increased interest in phenolic acids as components of the human diet, due to their health-promoting and antioxidant properties. Terry et al. (2014) demonstrated that phenolic compounds increased plant resistance to pathogens.

The popularity of biological crop protection agents containing bacteria and fungi, including members of the genus Trichoderma, is on the rise (Youssef et al. 2016). Biological protection agents have antibiotic and antiparasitic properties; they compete for nutrients with pathogens, induce systemic resistance and defense responses to the point of pathogen attack (biotic stress). In a study by Buysens et al. (2016), the application of T. harzianum and Rhizophagus irregularis to alfalfa (Medicago sativa) grown as a cover crop increased potato yields. Synthetic biostimulants (growth regulators, inorganic salts, phenolic compounds) and natural biostimulants (plant, bacterial and algal extracts) are also increasingly often used in agricultural practice (Tambascio et al. 2014). Algal extracts have complex composition and deliver various benefits, including greater yields (Wierzbowska et al. 2015) and higher resistance to abi- 
otic and biotic stress (Kosanić et al. 2015; Esserti et al. 2017). Commercial extracts of Ascophyllum nodosum (BioAlgeen S-90), Fucus spp., Laminaria spp., Sargassum spp. and Turbinaria spp. brown algae are most popular. The Vacciplant biological fungicide containing the extract of Laminaria digitata brown alga elicited defense responses in infected plants (Chojnacka et al. 2012). The spread of Pectobacterium carotovorum was effectively inhibited by acetone extracts of brown algae (Sargassum polyceratium) (Kumar et al. 2008) and methanol extracts of Padina gymnospora (Ibraheem et al. 2017). The Kelpak SL biostimulant containing the extract of Ecklonia maxima brown algae reduced the severity of black spot (Alternaria spp.) in rapeseed and Fusarium foot rot in cereals (Horoszkiewicz-Janka and Jajor 2006). Bio-Algeen S-90 Plus 2 improved the health status of barley, husked and naked oats (Horoszkiewicz-Janka and Michalski 2006). The extract of green algae of the genus Ulva protected common beans against Colletotrichum lindemithianum (Paulert et al. 2009), prevented the spread of Blumeria graminis in barley (Paulert et al. 2010), and the spread of powdery mildew (Erysiphe polygoni, E. necator and Sphaerotheca fuliginea) in beans, grapevines and cucumbers (Jaulneau et al. 2011). The effectiveness of biostimulants applied to plants exposed to stressors, as compared with greenhouse-grown plants, and their influence on plant health remains debatable (Calvo et al. 2014).

The aim of this study was to evaluate the influence of cultivar and biostimulants, important components of integrated crop protection systems, on the health status of potato tubers at harvest and after 5 months of storage and content of chlorogenic acids.

\section{Materials and Methods}

The experiment was performed on the tubers of five cultivars of edible potatoes grown in a micro-plot field experiment in the Agricultural Experiment Station in Tomaszkowo near Olsztyn $\left(53^{\circ} 41^{\prime} \mathrm{N}, \quad 20^{\circ} 24^{\prime} \mathrm{E}\right)$ in 2013-2015. The experiment had a randomized sub-block design with three replications. Two of the analyzed potato cultivars, Irga (cream-colored flesh) and Satina (yellowcolored flesh), are registered on the Polish National List of Agricultural Plant Varieties, and three cultivars, Valfi, Blaue St. Galler (blue-purple-colored flesh) and Highland Burgundy Red-HB Red (red-colored flesh), are listed in the European Common Catalogue of Varieties of Agricultural Plant Species. The soil, climate and agronomic conditions of the experiment have been described elsewhere (GłosekSobieraj et al. 2018). Seed potatoes were planted in heated soil, $40 \mathrm{~cm}$ apart, with inter-row spacing of $67.5 \mathrm{~cm}$. During the growing season, the following treatments were applied according to the manufacturers' recommendations:
- Asahi SL (0.1\% solution; natural nitrophenols:, $0.3 \%$ para-nitrophenol sodium salt, $0.2 \%$ ortho-nitrophenol sodium salt, $0.1 \%$ 5-nitroguaiacol sodium salt) (beginning from stage $\mathrm{BBCH} 39$; cover crop complete);

- Bio-Algeen S-90 (1\% solution; extract of Ascophyllum nodosum brown algae, amino acids, vitamins, alginic acid, $\mathrm{N}-0.2, \mathrm{P}_{2} \mathrm{O}_{5}-0.06, \mathrm{~K}_{2} \mathrm{O}-0.96, \mathrm{CaO}-3.1$, $\mathrm{MgO}-2.1 \mathrm{~g} \mathrm{~kg}^{-1}, \mathrm{~B}-16.0, \mathrm{Fe}-6.3, \mathrm{Cu}-0.2, \mathrm{Mn}-0.6$, $\mathrm{Zn}-1.0 \mathrm{mg} \mathrm{kg}^{-1}, \mathrm{Mo}, \mathrm{Se}, \mathrm{Co}$ - four foliar applications (beginning from stage BBCH 39);

- Kelpak SL $(0.2 \%$ solution; extract of Ecklonia maxima brown alga, $11 \mathrm{mg} \mathrm{dm}^{-3}$ auxins, $0.031 \mathrm{mg} \mathrm{dm}^{-3}$ cytokinins)—seed potato dressing and two foliar applications (beginning from stage BBCH 39);

- Trifender WP (Trichoderma asperellum, T1 isolate fungal spores at a concentration of $5 \times 10^{8} / \mathrm{g}$ of the product) - soil application and 4 foliar applications (beginning from stage $\mathrm{BBCH} 39$ ).

Control plants were not treated with the above biostimulants.

The severity of common scab (Streptomyces scabies) and black scurf (Rhizoctonia solani) was evaluated in 100 randomly sampled potato tubers at harvest and after 5 months of storage (at a temperature of $5^{\circ} \mathrm{C}$ ). The results of phytopathological evaluations were expressed in percentage by calculating the infection index according to the formula proposed by McKinney (Lacicowa 1970):

Infection index Ip $=\frac{\Sigma(\mathrm{a} \cdot \mathrm{b}) \cdot 100 \%}{\mathrm{~N} \cdot \mathrm{I}}$

where: $(a \cdot b)$ - sum of the products of the number of analyzed plants (a) and their severity scores (b), $N$-total number of analyzed plants, I-highest severity score.

The severity of soft rot (Pectobacterium carotovorum subsp. carotovorum, Dickeya spp.), late blight (Phytophthora infestans) and dry rot (Fusarium spp., Gibberella spp.) was evaluated in a $5 \mathrm{~kg}$ sample of potato tubers. The results were expressed by the percentage mass of infected tubers.

In a laboratory, fungal pathogens were isolated from potato tubers at harvest and after 5 months of storage. Cubes of potato flesh with skin, measuring $0.5 \times 0.5 \times 0.5 \mathrm{~cm}$, were cut out from 30 tubers (pooled sample from three microplots per treatment) from every experimental treatment. Potato cubes were disinfected with $50 \%$ ethanol and $0.1 \%$ sodium hypochlorite for $1 \mathrm{~min}$ each. They were rinsed three times in sterile water, dried on filter paper, placed on PDA (Sigma Aldrich) and incubated for 7 days in darkness at a temperature of $22^{\circ} \mathrm{C}$. Fungal colonies were identified.

Qualitative and quantitative analyses were performed by high-performance liquid chromatography (HPLC) using 
a photodiode array detector (PDA), coupled with mass spectrometry (Gliszczyńska et al. 2006).

The results were processed statistically by analysis of variance (ANOVA) in the Statistica 12.0 program. Group means were compared in Duncan's test at a significance level of 0.05 .

\section{Results and Discussion}

\section{Severity of Potato Diseases}

The average monthly temperatures between May and August 2013-2015 approximated the long-term average. In the analyzed months, total precipitation in the first growing season was close to the long-term average. In the following two growing seasons, total precipitation was 24 and $43 \%$ lower than the long-term average, respectively. The lowest precipitation was noted during tuber formation in 2014, which could have contributed to common scab infections. Weather conditions were not conducive to the spread of dry rot, soft rot, late blight and black scurf. In 2013, the volume and distribution of precipitation between the last ten days of June and the first twenty days of July could have contributed to the severity of the above infections in stored potatoes. The severity of common scab is determined by varietal susceptibility to disease, pathogen virulence, environmental conditions and the date of infection (Cullen and Lees 2007). According to Lenc (2006), the volume and distribution of precipitation in summer influence the severity of potato skin diseases. In this study, common scab symptoms were most severe on the tubers of the analyzed potato cultivars, particularly in 2014 , characterized by low precipitation levels during tuber formation. In the above year, Valfi and Blaue St. Galler tubers were infected in $75 \%$ immediately after harvest, whereas $70 \%$ of Valfi, Blaue St. Galler and Satina tubers were infected after 5 months of storage (Table 1). An analysis of the mean values noted in the examined cultivars and years indicates that potato cvs. Satina, Valfi and Blaue St. Galler were most severely infected $(60 \%)$ in the second year of the study, whereas in the remaining years of the experiment, the highest in-

Table 1 Infection of potato tubers by Streptomyces scabies (index of infection Ii \%)

\begin{tabular}{|c|c|c|c|c|c|c|c|c|c|c|c|c|c|}
\hline \multirow[t]{2}{*}{ Year } & & \multicolumn{6}{|c|}{ After harvest } & \multicolumn{6}{|c|}{ After storage } \\
\hline & & Irga & Satina & Valfi & Blaue & $\mathrm{HB}$ & $\mathrm{x}$ & Irga & Satina & Valfi & Blaue & $\mathrm{HB}$ & $\mathrm{x}$ \\
\hline \multirow[t]{5}{*}{2013} & $\bar{I}$ & $14.2^{0-x}$ & $31.3^{\mathrm{j}-\mathrm{m}}$ & $18.2^{\mathrm{n}-\mathrm{t}}$ & $17.3^{\mathrm{n}-\mathrm{v}}$ & $6.7^{\mathrm{s}-\mathrm{x}}$ & $17.5^{\mathrm{DE}}$ & $4.4^{t-x}$ & $35.1^{\mathrm{f}-\mathrm{l}}$ & $19.1^{1-u}$ & $16.0^{p-x}$ & $5.6^{\mathrm{s}-\mathrm{x}}$ & $16.0^{\mathrm{C}}$ \\
\hline & II & $13.3^{p-x}$ & $36.9^{\mathrm{ijk}}$ & $17.1^{\mathrm{n}-\mathrm{w}}$ & $10.2^{\mathrm{p}-\mathrm{x}}$ & $11.6^{\mathrm{p}-\mathrm{x}}$ & $17.8^{\mathrm{DE}}$ & $6.2^{\mathrm{s}-\mathrm{x}}$ & $31.3^{\mathrm{h}-\mathrm{p}}$ & $16.4^{p-x}$ & $10.9^{r-x}$ & $0.7^{\mathrm{x}}$ & $13.1^{\mathrm{C}}$ \\
\hline & III & $4.7^{\mathrm{u}-\mathrm{x}}$ & $40.9^{\mathrm{hij}}$ & $12.2^{p-x}$ & $3.3^{\mathrm{x}}$ & $3.6^{\mathrm{x}}$ & $12.9^{\mathrm{E}}$ & $0.7^{\mathrm{x}}$ & $25.3^{\mathrm{i}-\mathrm{r}}$ & $13.3^{g-x}$ & $5.1^{\mathrm{t}-\mathrm{x}}$ & $1.3^{\mathrm{wx}}$ & $9.1^{\mathrm{C}}$ \\
\hline & IV & $8.0^{\mathrm{s}-\mathrm{x}}$ & $40.7^{\text {hij }}$ & $17.1^{\mathrm{n}-\mathrm{w}}$ & $2.7^{\mathrm{x}}$ & $10.0^{q-x}$ & $15.7^{\mathrm{DE}}$ & $2.2^{\mathrm{u}-\mathrm{x}}$ & $28.0^{\mathrm{h}-\mathrm{q}}$ & $11.6^{q-x}$ & $4.7^{\mathrm{t}-\mathrm{x}}$ & $0.7^{\mathrm{x}}$ & $9.4^{\mathrm{C}}$ \\
\hline & $\mathrm{V}$ & $8.4^{\mathrm{s}-\mathrm{x}}$ & $42.0^{g-j}$ & $17.3^{\mathrm{n}-\mathrm{v}}$ & $7.6^{s-x}$ & $8.7^{r-x}$ & $16.8^{\mathrm{DE}}$ & $5.8^{\mathrm{s}-\mathrm{x}}$ & $36.2^{-\mathrm{f} j}$ & $6.0^{s-x}$ & $2.7^{\mathrm{t}-\mathrm{x}}$ & $4.0^{\mathrm{t}-\mathrm{x}}$ & $10.9^{\mathrm{C}}$ \\
\hline \multirow[t]{5}{*}{2014} & I & $41.2^{\mathrm{hij}}$ & $66.4^{\mathrm{a}-\mathrm{d}}$ & $64.3^{\mathrm{a}-\mathrm{d}}$ & $59.0^{\text {cde }}$ & $40.2^{\text {hij }}$ & $54.2^{\mathrm{C}}$ & $34.6^{\mathrm{f}-\mathrm{m}}$ & $64.5^{\mathrm{ab}}$ & $49.2^{\mathrm{b}-\mathrm{g}}$ & $50.5^{\mathrm{b}-\mathrm{f}}$ & $14.0^{q-x}$ & $42.6^{\mathrm{B}}$ \\
\hline & II & $54.7^{\mathrm{def}}$ & $64.5^{\mathrm{a}-\mathrm{d}}$ & $68.3^{\mathrm{abc}}$ & $60.8^{\mathrm{b}-\mathrm{e}}$ & $45.5^{\mathrm{f}-\mathrm{i}}$ & $58.7^{\mathrm{AB}}$ & $35.3^{\mathrm{f}-1}$ & $63.9^{\mathrm{abc}}$ & $55.8^{\mathrm{a}-\mathrm{d}}$ & $54.1^{\mathrm{a}-\mathrm{e}}$ & $24.3^{\mathrm{i}-\mathrm{r}}$ & $46.7^{\mathrm{AB}}$ \\
\hline & III & $54.2^{\mathrm{d}-\mathrm{g}}$ & $67.5^{\mathrm{abc}}$ & $59.9^{\text {cde }}$ & $66.5^{\mathrm{a}-\mathrm{d}}$ & $42.6^{\mathrm{f}-\mathrm{j}}$ & $57.4^{\mathrm{B}}$ & $39.8^{\mathrm{e}-\mathrm{i}}$ & $64.5^{\mathrm{ab}}$ & $55.4^{\mathrm{a}-\mathrm{d}}$ & $48.3^{\mathrm{c}-\mathrm{g}}$ & $23.3^{\mathrm{j}-\mathrm{r}}$ & $46.3^{\mathrm{B}}$ \\
\hline & IV & $51.1^{\mathrm{e}-\mathrm{h}}$ & $67.6^{\mathrm{abc}}$ & $69.2^{\mathrm{abc}}$ & $73.1^{\mathrm{ab}}$ & $44.1^{\mathrm{f}-\mathrm{i}}$ & $61.0^{\mathrm{AB}}$ & $42.7^{\mathrm{d}-\mathrm{h}}$ & $58.2^{\mathrm{a}-\mathrm{d}}$ & $62.3^{a b c}$ & $65.1^{\mathrm{ab}}$ & $12.4^{q-x}$ & $48.1^{\mathrm{AB}}$ \\
\hline & $\mathrm{V}$ & $62.8^{\mathrm{a}-\mathrm{e}}$ & $58.5^{\text {cde }}$ & $74.1^{\mathrm{a}}$ & $75.0^{\mathrm{a}}$ & $45.3^{\mathrm{f}-\mathrm{i}}$ & $63.1^{\mathrm{A}}$ & $42.7^{\mathrm{d}-\mathrm{h}}$ & $68.4^{\mathrm{a}}$ & $66.8^{a}$ & $64.7^{\mathrm{ab}}$ & $22.1^{\mathrm{j}-\mathrm{s}}$ & $52.9^{\mathrm{A}}$ \\
\hline \multirow[t]{5}{*}{2015} & I & $3.4^{\mathrm{x}}$ & $42.4^{\mathrm{f}-\mathrm{j}}$ & $18.5^{\mathrm{n}-\mathrm{t}}$ & $18.6^{\mathrm{n}-\mathrm{t}}$ & $5.5^{t-x}$ & $17.6^{\mathrm{DE}}$ & $1.5^{\mathrm{vwx}}$ & $34.3^{\mathrm{g}-\mathrm{n}}$ & $19.1^{1-u}$ & $16.3^{p-x}$ & $2.4^{\mathrm{u}-\mathrm{x}}$ & $14.7^{\mathrm{C}}$ \\
\hline & II & $2.8^{x}$ & $35.6^{\mathrm{ijk}}$ & $26.5^{\mathrm{k}-\mathrm{o}}$ & $21.6^{\mathrm{m}-\mathrm{r}}$ & $6.7^{\mathrm{s}-\mathrm{x}}$ & $18.6^{\mathrm{D}}$ & $0.9^{x}$ & $11.7^{q-x}$ & $17.9^{0-x}$ & $18.6^{\mathrm{m}-\mathrm{v}}$ & $4.7^{\mathrm{t}-\mathrm{x}}$ & $10.8^{\mathrm{C}}$ \\
\hline & III & $4.2^{\mathrm{vwx}}$ & $46.7^{\mathrm{f}-\mathrm{i}}$ & $22.1^{\mathrm{m}-\mathrm{q}}$ & $18.2^{\mathrm{n}-\mathrm{t}}$ & $3.7^{x}$ & $19.0^{\mathrm{D}}$ & $2.4^{\mathrm{u}-\mathrm{x}}$ & $33.5^{\mathrm{g}-\mathrm{o}}$ & $13.9^{q-x}$ & $16.4^{p-x}$ & $3.5^{\mathrm{t}-\mathrm{x}}$ & $13.9^{\mathrm{C}}$ \\
\hline & IV & $3.2^{\mathrm{x}}$ & $45.7^{\mathrm{f}-\mathrm{i}}$ & $28.2^{\mathrm{k}-\mathrm{n}}$ & $18.1^{\mathrm{n}-\mathrm{t}}$ & $4.2^{\mathrm{wx}}$ & $19.9^{\mathrm{D}}$ & $2.7^{\mathrm{t}-\mathrm{x}}$ & $33.5^{\mathrm{g}-\mathrm{o}}$ & $19.6^{\mathrm{k}-\mathrm{t}}$ & $17.5^{0-x}$ & $2.8^{\mathrm{t}-\mathrm{x}}$ & $15.2^{\mathrm{C}}$ \\
\hline & $\mathrm{V}$ & $6.2^{\mathrm{t}-\mathrm{x}}$ & $34.7^{i-1}$ & $28.2^{\mathrm{k}-\mathrm{n}}$ & $19.6^{\mathrm{m}-\mathrm{s}}$ & $2.3^{x}$ & $18.2^{\mathrm{DE}}$ & $1.1^{\mathrm{wx}}$ & $35.6^{\mathrm{f}-\mathrm{k}}$ & $18.1^{\mathrm{n}-\mathrm{w}}$ & $15.6^{\mathrm{p}-\mathrm{x}}$ & $4.4^{\mathrm{t}-\mathrm{x}}$ & $15.0^{\mathrm{C}}$ \\
\hline \multicolumn{14}{|l|}{ Mean } \\
\hline \multicolumn{2}{|l|}{2013} & $9.7^{\mathrm{G}}$ & $38.4^{\mathrm{D}}$ & $16.4^{\mathrm{F}}$ & $8.2^{\mathrm{GH}}$ & $8.1^{\mathrm{GH}}$ & $16.2^{\mathrm{C}}$ & $3.9^{\mathrm{G}}$ & $31.2^{\mathrm{D}}$ & $13.3^{\mathrm{EF}}$ & $7.9^{\mathrm{FG}}$ & $2.5^{\mathrm{G}}$ & $11.7^{\mathrm{B}}$ \\
\hline \multicolumn{2}{|l|}{2014} & $52.8^{\mathrm{B}}$ & $64.9^{\mathrm{A}}$ & $67.2^{\mathrm{A}}$ & $66.9^{\mathrm{A}}$ & $43.6^{\mathrm{C}}$ & $58.2^{\mathrm{A}}$ & $39.0^{\mathrm{C}}$ & $63.9^{\mathrm{A}}$ & $57.9^{\mathrm{B}}$ & $56.5^{\mathrm{B}}$ & $19.2^{\mathrm{E}}$ & $47.3^{\mathrm{A}}$ \\
\hline \multicolumn{2}{|l|}{2015} & $4.0^{\mathrm{H}}$ & $41.0^{\mathrm{CD}}$ & $24.7^{\mathrm{E}}$ & $18.7^{\mathrm{F}}$ & $5.1^{\mathrm{H}}$ & $18.7^{\mathrm{B}}$ & $1.7^{\mathrm{G}}$ & $29.7^{\mathrm{D}}$ & $17.7^{\mathrm{E}}$ & $16.9^{\mathrm{E}}$ & $3.6^{\mathrm{G}}$ & $13.9^{\mathrm{B}}$ \\
\hline \multicolumn{14}{|c|}{ Mean for 2013-2015 } \\
\hline \multicolumn{2}{|l|}{ I } & $19.6^{\mathrm{K}}$ & $46.7^{\mathrm{A}}$ & $33.7^{\mathrm{C}-\mathrm{F}}$ & $30.7^{\mathrm{D}-\mathrm{G}}$ & $17.5^{\mathrm{JK}}$ & $28.6^{\mathrm{B}}$ & $13.5^{\mathrm{FGH}}$ & $44.6^{\mathrm{A}}$ & $29.1^{\mathrm{CD}}$ & $27.6^{\mathrm{CD}}$ & $7.3^{\mathrm{GH}}$ & $24.4^{\mathrm{A}}$ \\
\hline \multicolumn{2}{|l|}{ II } & $23.6^{\mathrm{HIJ}}$ & $45.7^{\mathrm{AB}}$ & $37.3^{\mathrm{CDE}}$ & $30.9^{\mathrm{EFG}}$ & $21.3^{\mathrm{HIJ}}$ & $31.7^{\mathrm{A}}$ & $14.1^{\mathrm{FGH}}$ & $35.6^{\mathrm{BC}}$ & $30.0^{\mathrm{CD}}$ & $27.9^{\mathrm{CD}}$ & $9.9^{\mathrm{FGH}}$ & $23.5^{\mathrm{A}}$ \\
\hline \multicolumn{2}{|l|}{ III } & $21.0^{\mathrm{IJ}}$ & $51.7^{\mathrm{A}}$ & $31.4^{\mathrm{D}-\mathrm{G}}$ & $29.3^{\mathrm{FGH}}$ & $16.6^{\mathrm{JK}}$ & $30.0^{\mathrm{AB}}$ & $14.3^{\mathrm{FG}}$ & $41.1^{\mathrm{AB}}$ & $27.5^{\mathrm{CD}}$ & $23.3^{\mathrm{DE}}$ & $9.4^{\mathrm{FGH}}$ & $23.1^{\mathrm{A}}$ \\
\hline \multicolumn{2}{|l|}{ IV } & $20.8^{\mathrm{IJ}}$ & $51.3^{\mathrm{A}}$ & $38.2^{\mathrm{CD}}$ & $31.3^{\mathrm{D}-\mathrm{G}}$ & $19.4^{\mathrm{IJK}}$ & $32.2^{\mathrm{A}}$ & $15.9^{\mathrm{EFG}}$ & $39.9^{\mathrm{AB}}$ & $31.2^{\mathrm{CD}}$ & $29.1^{\mathrm{CD}}$ & $5.3^{\mathrm{H}}$ & $24.3^{\mathrm{A}}$ \\
\hline \multicolumn{2}{|l|}{ V } & $25.8^{\mathrm{GHI}}$ & $45.1^{\mathrm{AB}}$ & $39.8^{\mathrm{BC}}$ & $34.0^{\mathrm{C}-\mathrm{F}}$ & $19.8^{\mathrm{J}-\mathrm{K}}$ & $32.9^{\mathrm{A}}$ & $16.5^{\mathrm{EF}}$ & $46.7^{\mathrm{A}}$ & $30.3^{\mathrm{CD}}$ & $27.7^{\mathrm{CD}}$ & $10.2^{\mathrm{FGH}}$ & $26.3^{\mathrm{A}}$ \\
\hline \multicolumn{2}{|l|}{$\mathrm{x}$} & $22.2^{\mathrm{D}}$ & $47.9^{\mathrm{A}}$ & $36.1^{\mathrm{B}}$ & $31.4^{\mathrm{C}}$ & $18.7^{\mathrm{D}}$ & - & $14.9^{\mathrm{C}}$ & $41.6^{\mathrm{A}}$ & $29.6^{\mathrm{B}}$ & $27.1^{\mathrm{B}}$ & $8.4^{\mathrm{D}}$ & - \\
\hline
\end{tabular}

Values denoted by the same letters for years do not differ significantly at 5\% error (Duncan's test)

I control, II Asahi SL, III Bio-Algeen S-90, IV Kelpak SL, V Trifender WP, Blaue Blaue St. Galler, HB HB Red 
Table 2 Infection of potato tubers by Rhizoctonia solani (index of infection Ii \%)

\begin{tabular}{|c|c|c|c|c|c|c|c|c|c|c|c|c|c|}
\hline \multirow[t]{2}{*}{ Year } & & \multicolumn{6}{|c|}{ After harvest } & \multicolumn{6}{|c|}{ After storage } \\
\hline & & Irga & Satina & Valfi & Blaue & $\mathrm{HB}$ & $\mathrm{x}$ & Irga & Satina & Valfi & Blaue & $\mathrm{HB}$ & $\mathrm{x}$ \\
\hline \multirow[t]{5}{*}{2013} & $\mathrm{I}$ & $2.7^{\mathrm{c}-\mathrm{h}}$ & $1.8^{\mathrm{e}-\mathrm{h}}$ & $1.3^{\mathrm{gh}}$ & $0.0^{\mathrm{h}}$ & $0.0^{\mathrm{h}}$ & $1.2^{\mathrm{C}}$ & $9.1^{\mathrm{b}-\mathrm{f}}$ & $1.3^{\mathrm{ijk}}$ & $3.1^{\mathrm{f}-\mathrm{k}}$ & $1.3^{\mathrm{ijk}}$ & $2.7^{\mathrm{g}-\mathrm{k}}$ & $3.5^{\mathrm{A}-\mathrm{D}}$ \\
\hline & II & $1.8^{\mathrm{e}-\mathrm{h}}$ & $0.0^{\mathrm{h}}$ & $2.7^{\mathrm{c}-\mathrm{h}}$ & $0.0^{\mathrm{h}}$ & $0.7^{\mathrm{h}}$ & $1.0^{\mathrm{C}}$ & $2.2^{\mathrm{g}-\mathrm{k}}$ & $0.7^{\mathrm{ijk}}$ & $2.0^{\mathrm{g}-\mathrm{k}}$ & $1.8^{\mathrm{g}-\mathrm{k}}$ & $5.6^{\mathrm{c}-\mathrm{k}}$ & $2.5^{\mathrm{CD}}$ \\
\hline & III & $4.2^{\mathrm{c}-\mathrm{h}}$ & $0.0^{\mathrm{h}}$ & $0.0^{\mathrm{h}}$ & $0.7^{\mathrm{h}}$ & $0.0^{\mathrm{h}}$ & $1.0^{\mathrm{C}}$ & $9.1^{\mathrm{b}-\mathrm{f}}$ & $6.7^{c-i}$ & $3.8^{\mathrm{d}-\mathrm{k}}$ & $2.7^{\mathrm{g}-\mathrm{k}}$ & $5.3^{\mathrm{c}-\mathrm{k}}$ & $5.5^{\mathrm{A}}$ \\
\hline & IV & $5.6^{\mathrm{c}-\mathrm{h}}$ & $0.0^{\mathrm{h}}$ & $0.0^{\mathrm{h}}$ & $0.0^{\mathrm{h}}$ & $0.7^{\mathrm{h}}$ & $1.3^{\mathrm{C}}$ & $4.7^{\mathrm{d}-\mathrm{k}}$ & $2.7^{\mathrm{g}-\mathrm{k}}$ & $7.8^{\mathrm{c}-\mathrm{g}}$ & $2.7^{\mathrm{g}-\mathrm{k}}$ & $7.6^{\mathrm{c}-\mathrm{h}}$ & $5.1^{\mathrm{AB}}$ \\
\hline & V & $4.7^{\mathrm{c}-\mathrm{h}}$ & $1.3^{\mathrm{gh}}$ & $0.0^{\mathrm{h}}$ & $0.0^{\mathrm{h}}$ & $0.7^{\mathrm{h}}$ & $1.3^{\mathrm{C}}$ & $4.7^{\mathrm{d}-\mathrm{k}}$ & $4.0^{\mathrm{d}-\mathrm{k}}$ & $3.3^{\mathrm{e}-\mathrm{k}}$ & $0.7^{\mathrm{ijk}}$ & $4.4^{\mathrm{d}-\mathrm{k}}$ & $3.4^{\mathrm{A}-\mathrm{D}}$ \\
\hline \multirow[t]{5}{*}{2014} & I & $3.8^{\mathrm{c}-\mathrm{h}}$ & $3.6^{\mathrm{c}-\mathrm{h}}$ & $0.2^{\mathrm{h}}$ & $0.3^{\mathrm{h}}$ & $1.0^{\mathrm{h}}$ & $1.8^{\mathrm{BC}}$ & $5.2^{\mathrm{c}-\mathrm{k}}$ & $4.2^{\mathrm{d}-\mathrm{k}}$ & $0.0^{\mathrm{k}}$ & $0.7^{\mathrm{ijk}}$ & $0.8^{\mathrm{ijk}}$ & $2.2^{\mathrm{D}}$ \\
\hline & II & $4.3^{\mathrm{c}-\mathrm{h}}$ & $2.9^{c-h}$ & $0.4^{\mathrm{h}}$ & $1.0^{\mathrm{h}}$ & $0.4^{\mathrm{h}}$ & $1.8^{\mathrm{BC}}$ & $11.0^{\mathrm{abc}}$ & $3.6^{\mathrm{d}-\mathrm{k}}$ & $0.0^{\mathrm{k}}$ & $0.0^{\mathrm{k}}$ & $0.5^{\mathrm{ijk}}$ & $3.0^{\mathrm{A}-\mathrm{D}}$ \\
\hline & III & $4.5^{\mathrm{c}-\mathrm{h}}$ & $1.6^{\mathrm{fgh}}$ & $0.0^{\mathrm{h}}$ & $0.0^{\mathrm{h}}$ & $0.0^{\mathrm{h}}$ & $1.2^{\mathrm{C}}$ & $4.5^{\mathrm{d}-\mathrm{k}}$ & $4.1^{\mathrm{d}-\mathrm{k}}$ & $0.0^{\mathrm{k}}$ & $0.0^{\mathrm{k}}$ & $0.8^{\mathrm{ijk}}$ & $1.9^{\mathrm{D}}$ \\
\hline & IV & $7.3^{\mathrm{b}-\mathrm{f}}$ & $7.4^{\mathrm{b}-\mathrm{e}}$ & $0.0^{\mathrm{h}}$ & $0.4^{\mathrm{h}}$ & $0.4^{\mathrm{h}}$ & $3.1^{\mathrm{ABC}}$ & $4.3^{\mathrm{d}-\mathrm{k}}$ & $3.8^{\mathrm{d}-\mathrm{k}}$ & $0.2^{\mathrm{jk}}$ & $0.4^{\mathrm{ijk}}$ & $0.4^{\mathrm{ijk}}$ & $1.8^{\mathrm{D}}$ \\
\hline & $\mathrm{V}$ & $8.1^{\mathrm{bc}}$ & $3.4^{\mathrm{c}-\mathrm{h}}$ & $0.0^{\mathrm{h}}$ & $0.7^{\mathrm{h}}$ & $0.0^{\mathrm{h}}$ & $2.4^{\mathrm{BC}}$ & $9.4^{\mathrm{b}-\mathrm{e}}$ & $5.5^{\mathrm{c}-\mathrm{k}}$ & $0.0^{\mathrm{k}}$ & $0.0^{\mathrm{k}}$ & $0.0^{\mathrm{k}}$ & $3.0^{\mathrm{BCD}}$ \\
\hline \multirow[t]{5}{*}{2015} & I & $11.0^{\mathrm{ab}}$ & $5.4^{\mathrm{c}-\mathrm{h}}$ & $1.8^{\mathrm{e}-\mathrm{h}}$ & $0.7^{\mathrm{h}}$ & $0.5^{\mathrm{h}}$ & $3.9^{\mathrm{AB}}$ & $14.8^{\mathrm{a}}$ & $6.4^{\mathrm{c}-\mathrm{j}}$ & $1.1^{\mathrm{ijk}}$ & $0.2^{\mathrm{jk}}$ & $1.5^{\mathrm{h}-\mathrm{k}}$ & $4.8^{\mathrm{ABC}}$ \\
\hline & II & $3.3^{\mathrm{c}-\mathrm{h}}$ & $1.6^{\mathrm{fgh}}$ & $1.1^{\mathrm{h}}$ & $2.5^{\mathrm{c}-\mathrm{h}}$ & $0.0^{\mathrm{h}}$ & $1.7^{\mathrm{BC}}$ & $3.0^{\mathrm{f}-\mathrm{k}}$ & $2.0^{\mathrm{g}-\mathrm{k}}$ & $0.9^{\mathrm{ijk}}$ & $0.0^{\mathrm{k}}$ & $1.7^{\mathrm{g}-\mathrm{k}}$ & $1.5^{\mathrm{D}}$ \\
\hline & III & $6.9^{\mathrm{b}-\mathrm{g}}$ & $2.2^{\mathrm{d}-\mathrm{h}}$ & $1.5^{\mathrm{gh}}$ & $1.6^{\mathrm{fgh}}$ & $0.2^{\mathrm{h}}$ & $2.5^{\mathrm{BC}}$ & $9.5^{\mathrm{bcd}}$ & $2.4^{\mathrm{g}-\mathrm{k}}$ & $0.4^{\mathrm{ijk}}$ & $4.3^{\mathrm{d}-\mathrm{k}}$ & $2.0^{\mathrm{g}-\mathrm{k}}$ & $3.7^{\mathrm{A}-\mathrm{D}}$ \\
\hline & IV & $7.7^{\mathrm{bcd}}$ & $0.5^{\mathrm{h}}$ & $0.4^{\mathrm{h}}$ & $1.0^{\mathrm{h}}$ & $0.0^{\mathrm{h}}$ & $1.9^{\mathrm{BC}}$ & $6.7^{\mathrm{c}-\mathrm{i}}$ & $2.3^{\mathrm{g}-\mathrm{k}}$ & $0.4^{\mathrm{ijk}}$ & $0.2^{\mathrm{jk}}$ & $1.3^{\mathrm{ijk}}$ & $2.2^{\mathrm{D}}$ \\
\hline & $\mathrm{V}$ & $14.1^{\mathrm{a}}$ & $5.0^{\mathrm{c}-\mathrm{h}}$ & $4.8^{\mathrm{c}-\mathrm{h}}$ & $0.7^{\mathrm{h}}$ & $0.4^{\mathrm{h}}$ & $5.0^{\mathrm{A}}$ & $13.5^{\mathrm{ab}}$ & $3.2^{\mathrm{f}-\mathrm{k}}$ & $1.6^{\mathrm{g}-\mathrm{k}}$ & $0.8^{\mathrm{ijk}}$ & $1.1^{\mathrm{ijk}}$ & $4.1^{\mathrm{A}-\mathrm{D}}$ \\
\hline \multicolumn{14}{|l|}{ Mean } \\
\hline \multicolumn{2}{|l|}{2013} & $3.8^{\mathrm{BC}}$ & $0.6^{\mathrm{E}}$ & $0.8^{\mathrm{DE}}$ & $0.1^{\mathrm{E}}$ & $0.4^{\mathrm{E}}$ & $1.2^{\mathrm{B}}$ & $6.0^{\mathrm{BC}}$ & $3.1^{\mathrm{DEF}}$ & $4.0^{\mathrm{CDE}}$ & $1.8^{\mathrm{EFG}}$ & $5.1^{\mathrm{BCD}}$ & $4.0^{\mathrm{A}}$ \\
\hline \multicolumn{2}{|l|}{2014} & $5.6^{\mathrm{B}}$ & $3.8^{\mathrm{BC}}$ & $0.1^{\mathrm{E}}$ & $0.5^{\mathrm{E}}$ & $0.3^{\mathrm{E}}$ & $2.1^{\mathrm{B}}$ & $6.9^{\mathrm{B}}$ & $4.2^{\mathrm{CDE}}$ & $0.0^{\mathrm{G}}$ & $0.2^{\mathrm{G}}$ & $0.5^{\mathrm{G}}$ & $2.4^{\mathrm{B}}$ \\
\hline \multicolumn{2}{|l|}{2015} & $8.6^{\mathrm{A}}$ & $3.0^{\mathrm{CD}}$ & $1.9^{\mathrm{CDE}}$ & $1.3^{\mathrm{DE}}$ & $0.2^{\mathrm{E}}$ & $3.0^{\mathrm{A}}$ & $9.5^{\mathrm{A}}$ & $3.3^{\mathrm{DEF}}$ & $0.9^{\mathrm{FG}}$ & $1.1^{\mathrm{FG}}$ & $1.5^{\mathrm{FG}}$ & $3.3^{\mathrm{AB}}$ \\
\hline \multicolumn{14}{|c|}{ Mean for 2013-2015 } \\
\hline \multicolumn{2}{|l|}{ I } & $5.8^{\mathrm{BC}}$ & $3.6^{\mathrm{CDE}}$ & $1.1^{\mathrm{EF}}$ & $0.3^{\mathrm{F}}$ & $0.5^{\mathrm{EF}}$ & $2.3^{\mathrm{AB}}$ & $9.7^{\mathrm{A}}$ & $4.0^{\mathrm{C}-\mathrm{F}}$ & $1.4^{\mathrm{D}-\mathrm{G}}$ & $0.7^{\mathrm{FG}}$ & $1.7^{\mathrm{D}-\mathrm{G}}$ & $3.5^{\mathrm{A}}$ \\
\hline \multicolumn{2}{|l|}{ II } & $3.1^{\mathrm{C}-\mathrm{F}}$ & $1.5^{\mathrm{EF}}$ & $1.4^{\mathrm{EF}}$ & $1.2^{\mathrm{EF}}$ & $0.4^{\mathrm{EF}}$ & $1.5^{\mathrm{B}}$ & $5.4^{\mathrm{BC}}$ & $2.1^{\mathrm{C}-\mathrm{G}}$ & $1.0^{\mathrm{EFG}}$ & $0.6^{\mathrm{FG}}$ & $2.6^{\mathrm{C}-\mathrm{G}}$ & $2.3^{\mathrm{A}}$ \\
\hline \multicolumn{2}{|l|}{ III } & $5.2^{\mathrm{BCD}}$ & $1.3^{\mathrm{EF}}$ & $0.5^{\mathrm{EF}}$ & $0.8^{\mathrm{EF}}$ & $0.1^{\mathrm{F}}$ & $1.6^{\mathrm{B}}$ & $7.7^{\mathrm{AB}}$ & $4.4^{\mathrm{BC}}$ & $1.4^{\mathrm{D}-\mathrm{G}}$ & $2.3^{\mathrm{C}-\mathrm{G}}$ & $2.7^{\mathrm{C}-\mathrm{G}}$ & $3.7^{\mathrm{A}}$ \\
\hline \multicolumn{2}{|l|}{ IV } & $6.9^{\mathrm{AB}}$ & $2.6^{\mathrm{DEF}}$ & $0.1^{\mathrm{F}}$ & $0.5^{\mathrm{EF}}$ & $0.4^{\mathrm{EF}}$ & $2.1^{\mathrm{AB}}$ & $5.2^{\mathrm{BC}}$ & $2.9^{\mathrm{C}-\mathrm{G}}$ & $2.8^{\mathrm{C}-\mathrm{G}}$ & $1.1^{\mathrm{D}-\mathrm{G}}$ & $3.1^{\mathrm{C}-\mathrm{G}}$ & $3.0^{\mathrm{A}}$ \\
\hline \multicolumn{2}{|l|}{ V } & $8.9^{\mathrm{A}}$ & $3.2^{\mathrm{C}-\mathrm{F}}$ & $1.6^{\mathrm{EF}}$ & $0.5^{\mathrm{EF}}$ & $0.4^{\mathrm{EF}}$ & $2.9^{\mathrm{A}}$ & $9.2^{\mathrm{A}}$ & $4.2^{\mathrm{CDE}}$ & $1.6^{\mathrm{D}-\mathrm{G}}$ & $0.5^{\mathrm{G}}$ & $1.8^{\mathrm{D}-\mathrm{G}}$ & $3.5^{\mathrm{A}}$ \\
\hline \multicolumn{2}{|l|}{$\mathrm{x}$} & $6.0^{\mathrm{A}}$ & $2.5^{\mathrm{B}}$ & $0.9^{\mathrm{C}}$ & $0.6^{\mathrm{C}}$ & $0.3^{\mathrm{C}}$ & - & $7.4^{\mathrm{A}}$ & $3.5^{\mathrm{B}}$ & $1.6^{\mathrm{CD}}$ & $1.1^{\mathrm{D}}$ & $2.4^{\mathrm{BC}}$ & - \\
\hline
\end{tabular}

Values denoted by the same letters for years do not differ significantly at 5\% error (Duncan's test)

I control, II Asahi SL, III Bio-Algeen S-90, IV Kelpak SL, V Trifender WP, Blaue Blaue St. Galler, HB HB Red

fection rate $(40 \%)$ was observed in tubers cv. Satina which differed significantly from the remaining cultivars. The least infected cultivars were Irga and HB Red in 2014 as well as Blaue St. Galler in 2013. The mean values noted in the examined cultivars and treatments indicate that biostimulants significantly intensified the symptoms of common scab in potatoes $\mathrm{cv}$. Irga, but did not induce significant differences in disease severity between the remaining cultivars and treatments. The severity of common scab did not increase with storage time. Bacterial infections were most severe in tubers cv. Satina harvested in successive years of the study. In the above cultivar, disease symptoms were significantly reduced only in the treatment with the Asahi SL biostimulant. The applied biostimulants did not induce significant differences in the severity of the disease in the remaining cultivars. According to Sawicka (1999), growth regulators (Mival, Potejtin, Moddus 250 ME) intensified the severity of Streptomyces scabies infections, in particular in dry years. 2,4-dichlorophenoxyacetic acid (2,4-D) reduced the symptoms of the disease caused by S. scabies (Waterer
2010) and influenced the growth of potato plants, potato yields and the color of red-skinned cultivars (Red LaSoda and TerraRosa) (Thornton et al. 2013). In a previous study by Cwalina-Ambroziak et al. (2015), Bio-Algeen S-90 and Kelpak SL growth regulators and the Asahi SL biostimulant did not affect the severity of common scab, soft rot and black scurf on stored potato tubers.

Bains et al. (2002) reported differences in the incidence and severity of black scurf between potato cultivars. Lutomirska (2008) observed such differences between experimental years and concluded that high soil moisture content and low ambient temperature contributed to the spread of $R$. solani. In this study, the infection index was highest in 2015 (with evenly distributed precipitation in July) in potatoes cv. Irga grown without and with the Trifender WP biostimulant, at 11 and $14 \%$ at harvest, and at 14.8 and $13.5 \%$ after 5 months of storage, respectively (Table 2). An analysis of the mean values revealed that freshly harvested potatoes cv. Irga were most severely infected with Rhizoctonia solani (highest infection index of $8.6 \%$ in 2015) in suc- 
Table 3 Infection of potato tubers by Fusarium spp. (\% of the total weight of infected tubers)

\begin{tabular}{|c|c|c|c|c|c|c|c|c|c|c|c|c|c|}
\hline \multirow[t]{2}{*}{ Year } & & \multicolumn{6}{|c|}{ After harvest } & \multicolumn{6}{|c|}{ After storage } \\
\hline & & Irga & Satina & Valfi & Blaue & $\mathrm{HB}$ & $\mathrm{x}$ & Irga & Satina & Valfi & Blaue & $\mathrm{HB}$ & $\mathrm{x}$ \\
\hline \multirow[t]{5}{*}{2013} & I & $0.0^{1}$ & $3.7^{\mathrm{d}}$ & $0.0^{1}$ & $0.0^{1}$ & $0.6^{\mathrm{h}-\mathrm{l}}$ & $0.9^{\mathrm{CD}}$ & $21.6^{\mathrm{b}}$ & $10.5^{\text {efg }}$ & $6.7^{\mathrm{h}-1}$ & $11.1^{\mathrm{d}-\mathrm{g}}$ & $5.7^{j-n}$ & $11.1^{\mathrm{C}}$ \\
\hline & II & $0.0^{1}$ & $0.0^{1}$ & $0.0^{1}$ & $0.0^{1}$ & $0.0^{1}$ & $0.0^{\mathrm{G}}$ & $22.4^{\mathrm{b}}$ & $15.4^{\mathrm{c}}$ & $12.7^{\mathrm{c}-\mathrm{f}}$ & $5.1^{\mathrm{j}-\mathrm{q}}$ & $5.4^{\mathrm{j}-\mathrm{p}}$ & $12.2^{\mathrm{BC}}$ \\
\hline & III & $6.4^{\mathrm{b}}$ & $0.0^{1}$ & $2.1^{\mathrm{efg}}$ & $1.1^{\mathrm{h}-\mathrm{l}}$ & $0.0^{1}$ & $1.9^{\mathrm{B}}$ & $21.4^{\mathrm{b}}$ & $11.0^{\mathrm{d}-\mathrm{g}}$ & $15.9^{c}$ & $6.8^{\mathrm{h}-\mathrm{k}}$ & $9.5^{\mathrm{f}-\mathrm{i}}$ & $12.9^{\mathrm{AB}}$ \\
\hline & IV & $13.0^{\mathrm{a}}$ & $0.0^{1}$ & $2.6^{\mathrm{e}}$ & $0.0^{1}$ & $0.0^{1}$ & $3.1^{\mathrm{A}}$ & $20.8^{\mathrm{b}}$ & $23.7^{\mathrm{b}}$ & $5.0^{\mathrm{j}-\mathrm{q}}$ & $5.1^{\mathrm{j}-\mathrm{q}}$ & $4.0^{\mathrm{k}-\mathrm{t}}$ & $11.7^{\mathrm{BC}}$ \\
\hline & V & $5.2^{\mathrm{c}}$ & $0.0^{1}$ & $0.0^{1}$ & $0.0^{1}$ & $0.7^{\mathrm{h}-1}$ & $1.2^{\mathrm{C}}$ & $27.7^{\mathrm{a}}$ & $13.5^{\text {cde }}$ & $9.8^{\mathrm{fgh}}$ & $14.2^{\mathrm{cd}}$ & $3.9^{\mathrm{k}-\mathrm{t}}$ & $13.8^{\mathrm{A}}$ \\
\hline \multirow[t]{5}{*}{2014} & I & $0.0^{1}$ & $1.4^{\mathrm{f}-\mathrm{j}}$ & $2.4^{\mathrm{ef}}$ & $0.7^{\mathrm{h}-\mathrm{l}}$ & $0.6^{\mathrm{h}-\mathrm{l}}$ & $1.0^{\mathrm{CD}}$ & $2.7^{\mathrm{m}-\mathrm{t}}$ & $1.5^{\mathrm{p}-\mathrm{t}}$ & $2.6^{\mathrm{m}-\mathrm{t}}$ & $1.3^{\mathrm{q}-\mathrm{t}}$ & $2.2^{\mathrm{m}-\mathrm{t}}$ & $2.1^{\mathrm{DE}}$ \\
\hline & II & $0.0^{1}$ & $0.0^{1}$ & $1.5^{\mathrm{e}-\mathrm{h}}$ & $1.4^{\mathrm{f}-\mathrm{j}}$ & $0.3^{\mathrm{i}-1}$ & $0.7^{\mathrm{DE}}$ & $1.2^{\mathrm{q}-\mathrm{t}}$ & $0.0^{\mathrm{t}}$ & $2.2^{\mathrm{m}-\mathrm{t}}$ & $1.4^{\mathrm{q}-\mathrm{t}}$ & $2.0^{\mathrm{n}-\mathrm{t}}$ & $1.4^{\mathrm{E}}$ \\
\hline & III & $0.0^{1}$ & $0.3^{\mathrm{i}-1}$ & $0.2^{\mathrm{kl}}$ & $0.0^{1}$ & $1.2^{\mathrm{g}-\mathrm{l}}$ & $0.3^{\mathrm{EFG}}$ & $0.8^{\mathrm{rst}}$ & $3.3^{\mathrm{k}-\mathrm{t}}$ & $2.1^{\mathrm{n}-\mathrm{t}}$ & $2.1^{\mathrm{n}-\mathrm{t}}$ & $1.2^{\mathrm{q}-\mathrm{t}}$ & $1.9^{\mathrm{DE}}$ \\
\hline & IV & $0.0^{1}$ & $1.3^{\mathrm{g}-\mathrm{k}}$ & $0.9^{\mathrm{h}-\mathrm{l}}$ & $2.0^{\text {efg }}$ & $0.7^{\mathrm{h}-1}$ & $1.0^{\mathrm{CD}}$ & $4.9^{\mathrm{j}-\mathrm{q}}$ & $4.0^{\mathrm{k}-\mathrm{t}}$ & $3.7^{\mathrm{k}-\mathrm{t}}$ & $0.5^{\mathrm{st}}$ & $2.9^{1-t}$ & $3.2^{\mathrm{D}}$ \\
\hline & V & $0.0^{1}$ & $0.0^{\mathrm{l}}$ & $0.4^{\mathrm{h}-\mathrm{l}}$ & $1.6^{\mathrm{e}-\mathrm{h}}$ & $0.9^{\mathrm{h}-\mathrm{l}}$ & $0.6^{\mathrm{DEF}}$ & $2.1^{\mathrm{n}-\mathrm{t}}$ & $2.1^{\mathrm{n}-\mathrm{t}}$ & $2.9^{1-t}$ & $2.5^{\mathrm{m}-\mathrm{t}}$ & $3.0^{\mathrm{k}-\mathrm{t}}$ & $2.5^{\mathrm{DE}}$ \\
\hline \multirow[t]{5}{*}{2015} & I & $0.2^{\mathrm{kl}}$ & $0.5^{\mathrm{h}-\mathrm{l}}$ & $0.1^{\mathrm{kl}}$ & $0.1^{\mathrm{kl}}$ & $0.0^{1}$ & $0.2^{\mathrm{FG}}$ & $3.7^{\mathrm{k}-\mathrm{t}}$ & $5.1^{\mathrm{j}-\mathrm{q}}$ & $1.6^{\mathrm{o}-\mathrm{t}}$ & $2.2^{\mathrm{n}-\mathrm{t}}$ & $0.7^{\mathrm{rst}}$ & $2.7^{\mathrm{DE}}$ \\
\hline & II & $0.1^{\mathrm{kl}}$ & $0.1^{\mathrm{kl}}$ & $0.0^{1}$ & $0.1^{\mathrm{kl}}$ & $0.0^{1}$ & $0.1^{\mathrm{G}}$ & $6.1^{\mathrm{i}-\mathrm{m}}$ & $4.5^{\mathrm{j}-\mathrm{r}}$ & $0.4^{\mathrm{st}}$ & $3.5^{\mathrm{k}-\mathrm{t}}$ & $1.3^{\mathrm{q}-\mathrm{t}}$ & $3.2^{\mathrm{D}}$ \\
\hline & III & $0.2^{\mathrm{kl}}$ & $0.2^{\mathrm{kl}}$ & $0.1^{\mathrm{kl}}$ & $0.1^{\mathrm{kl}}$ & $0.0^{1}$ & $0.1^{\mathrm{G}}$ & $2.9^{\mathrm{l}-\mathrm{t}}$ & $7.8^{\mathrm{g}-\mathrm{j}}$ & $1.5^{\mathrm{p}-\mathrm{t}}$ & $2.3^{\mathrm{m}-\mathrm{t}}$ & $1.9^{\mathrm{n}-\mathrm{t}}$ & $3.3^{\mathrm{D}}$ \\
\hline & IV & $0.1^{\mathrm{kl}}$ & $0.1^{\mathrm{kl}}$ & $0.1^{\mathrm{kl}}$ & $0.1^{\mathrm{kl}}$ & $0.1^{\mathrm{kl}}$ & $0.1^{\mathrm{G}}$ & $1.4^{\mathrm{q}-\mathrm{t}}$ & $2.5^{\mathrm{m}-\mathrm{t}}$ & $2.2^{\mathrm{n}-\mathrm{t}}$ & $1.3^{\mathrm{q}-\mathrm{t}}$ & $2.4^{\mathrm{m}-\mathrm{t}}$ & $1.9^{\mathrm{DE}}$ \\
\hline & $\mathrm{V}$ & $0.2^{\mathrm{kl}}$ & $0.2^{\mathrm{kl}}$ & $0.1^{\mathrm{kl}}$ & $0.0^{1}$ & $0.1^{\mathrm{kl}}$ & $0.1^{\mathrm{G}}$ & $5.6^{\mathrm{j}-\mathrm{o}}$ & $4.8^{\mathrm{j}-\mathrm{q}}$ & $0.5^{\mathrm{st}}$ & $4.0^{\mathrm{k}-\mathrm{t}}$ & $1.5^{\mathrm{p}-\mathrm{t}}$ & $3.3^{\mathrm{D}}$ \\
\hline \multicolumn{14}{|l|}{ Mean } \\
\hline \multicolumn{2}{|l|}{2013} & $4.9^{\mathrm{A}}$ & $0.7^{\mathrm{BC}}$ & $0.9^{\mathrm{BC}}$ & $0.2^{\mathrm{DE}}$ & $0.3^{\mathrm{DE}}$ & $1.4^{\mathrm{A}}$ & $22.8^{\mathrm{A}}$ & $14.8^{\mathrm{B}}$ & $10.0^{\mathrm{C}}$ & $8.5^{\mathrm{D}}$ & $5.7^{\mathrm{E}}$ & $12.4^{\mathrm{A}}$ \\
\hline \multicolumn{2}{|l|}{2014} & $0.0^{\mathrm{E}}$ & $0.6^{\mathrm{CD}}$ & $1.1^{\mathrm{B}}$ & $1.1^{\mathrm{B}}$ & $0.7^{\mathrm{BC}}$ & $0.7^{\mathrm{B}}$ & $2.3^{\mathrm{H}}$ & $2.2^{\mathrm{H}}$ & $2.7^{\mathrm{GH}}$ & $1.6^{\mathrm{H}}$ & $2.3^{\mathrm{H}}$ & $2.2^{\mathrm{C}}$ \\
\hline \multicolumn{2}{|l|}{2015} & $0.1^{\mathrm{E}}$ & $0.2^{\mathrm{DE}}$ & $0.1^{\mathrm{E}}$ & $0.1^{\mathrm{E}}$ & $0.0^{\mathrm{E}}$ & $0.1^{\mathrm{C}}$ & $3.9^{\mathrm{FG}}$ & $4.9^{\mathrm{EF}}$ & $1.2^{\mathrm{H}}$ & $2.6^{\mathrm{GH}}$ & $1.6^{\mathrm{H}}$ & $2.9^{\mathrm{B}}$ \\
\hline \multicolumn{14}{|c|}{ Mean for 2013-2015 } \\
\hline \multicolumn{2}{|l|}{ I } & $0.1^{\mathrm{E}}$ & $1.9^{\mathrm{B}}$ & $0.9^{\mathrm{CD}}$ & $0.3^{\mathrm{DE}}$ & $0.4^{\mathrm{DE}}$ & $0.7^{\mathrm{B}}$ & $9.3^{\mathrm{B}}$ & $5.7^{\mathrm{EFG}}$ & $3.6^{\mathrm{HIJ}}$ & $4.9^{\mathrm{F}-\mathrm{I}}$ & $2.9^{\mathrm{IJ}}$ & $5.3^{\mathrm{B}}$ \\
\hline \multicolumn{2}{|l|}{ II } & $0.0^{\mathrm{E}}$ & $0.0^{\mathrm{E}}$ & $0.5^{\mathrm{DE}}$ & $0.5^{\mathrm{DE}}$ & $0.1^{\mathrm{E}}$ & $0.2^{\mathrm{C}}$ & $9.9^{\mathrm{B}}$ & $6.6^{\mathrm{DEF}}$ & $5.1^{\mathrm{FGH}}$ & $3.3^{\mathrm{HIJ}}$ & $2.9^{\mathrm{IJ}}$ & $5.6^{\mathrm{B}}$ \\
\hline \multicolumn{2}{|l|}{ III } & $2.2^{\mathrm{B}}$ & $0.2^{\mathrm{DE}}$ & $0.8^{\mathrm{CD}}$ & $0.4^{\mathrm{DE}}$ & $0.4^{\mathrm{DE}}$ & $0.8^{\mathrm{B}}$ & $8.4^{\mathrm{CD}}$ & $7.4^{\mathrm{CDE}}$ & $6.5^{\mathrm{DEF}}$ & $3.8^{\mathrm{G}-\mathrm{J}}$ & $4.2^{\mathrm{G}-\mathrm{J}}$ & $6.0^{\mathrm{AB}}$ \\
\hline \multicolumn{2}{|l|}{ IV } & $4.4^{\mathrm{A}}$ & $0.5^{\mathrm{DE}}$ & $1.2^{\mathrm{C}}$ & $0.7^{\mathrm{CDE}}$ & $0.2^{\mathrm{DE}}$ & $1.4^{\mathrm{A}}$ & $9.0^{\mathrm{BC}}$ & $10.1^{\mathrm{AB}}$ & $3.6^{\mathrm{HIJ}}$ & $2.3^{\mathrm{J}}$ & $3.1^{\mathrm{HIJ}}$ & $5.6^{\mathrm{B}}$ \\
\hline \multicolumn{2}{|l|}{ V } & $1.8^{\mathrm{B}}$ & $0.1^{\mathrm{E}}$ & $0.2^{\mathrm{DE}}$ & $0.5^{\mathrm{DE}}$ & $0.5^{\mathrm{DE}}$ & $0.6^{\mathrm{B}}$ & $11.8^{\mathrm{A}}$ & $6.8^{\mathrm{DEF}}$ & $4.4^{\mathrm{G}-\mathrm{J}}$ & $6.9^{\mathrm{DEF}}$ & $2.8^{\mathrm{IJ}}$ & $6.5^{\mathrm{A}}$ \\
\hline \multicolumn{2}{|l|}{$\mathrm{x}$} & $1.7^{\mathrm{A}}$ & $0.5^{\mathrm{BC}}$ & $0.7^{\mathrm{B}}$ & $0.5^{\mathrm{BC}}$ & $0.3^{\mathrm{C}}$ & - & $9.7^{\mathrm{A}}$ & $7.3^{\mathrm{B}}$ & $4.7^{\mathrm{C}}$ & $4.2^{\mathrm{C}}$ & $3.2^{\mathrm{D}}$ & - \\
\hline
\end{tabular}

Values denoted by the same letters for years do not differ significantly at 5\% error (Duncan's test)

I control, II Asahi SL, III Bio-Algeen S-90, IV Kelpak SL, V Trifender WP, Blaue Blaue St. Galler, HB HB Red

cessive years of the experiment, and these results differed significantly from those noted in the remaining cultivars. In 2013, less than $2 \%$ of Valfi, Blaue St. Galler, HB Red and Satina potatoes were infected. The applied biostimulants limited the severity of black scurf in tubers cv. Satina, but the observed differences were not significant relative to the control treatment. A significant increase in the severity of black scurf was noted in potatoes cv. Irga treated with the Trifender WP biostimulant. The above observations contradict the results of published studies where Trichoderma spp. species were more effective antagonists of $R$. solani in infected than in healthy potatoes (Anees et al. 2010), and in soils with a higher abundance of sclerotia (Grosch et al. 2006). In potatoes cvs. Valfi, Blaue St. Galler and HB Red, the prevalence of black scurf was similar at harvest and after 5 months of storage. Asahi SL and Kelpak SL reduced the severity of the disease in tubers cv. Irga and Satina, but the observed difference was significant relative to the control treatment only in cv. Irga. The applied biostimulants exerted varied effects on the remaining cul- tivars. The extracts of Stokeyia indica and Padina pavonia brown algae and Solieria robusta red alga were effective in protecting Abelmoschus esculentus against Rhizoctonia solani (Sultana et al. 2005). Raj et al. (2016) reported on the fungicidal effects of the extract of Sargassum swartzii brown algae which limited the spread of Rhizoctonia solani infections in rice. According to the above authors, the algal extract stimulated a defense response in rice plants by inducing phenols and early accumulation of phytoalexins. In a previous study by Cwalina-Ambroziak et al. (2015), potatoes cvs. Irga and Satina were most severely infected with $R$. solani $(7 \%)$ in the treatment with the Asahi SL biostimulant. In the cited experiment, Bio-Algeen S-90 and Kelpak SL growth regulators and the Asahi SL biostimulant exerted varied effects on the spread of black scurf in stored tubers cvs. Irga and Satina.

The symptoms of soft rot were observed sporadically at harvest and after 5 months of storage and dry rot and late blight at harvest in all experimental years. Low precipitation levels in May, June and early July (especially in the last 
Table 4 Infection of potato tubers by Phytophthora infestans (\% of the total weight of infected tubers)

\begin{tabular}{|c|c|c|c|c|c|c|c|c|c|c|c|c|c|}
\hline \multirow[t]{2}{*}{ Year } & & \multicolumn{6}{|c|}{ After harvest } & \multicolumn{6}{|c|}{ After storage } \\
\hline & & Irga & Satina & Valfi & Blaue & $\mathrm{HB}$ & $\mathrm{x}$ & Irga & Satina & Valfi & Blaue & $\mathrm{HB}$ & $\mathrm{x}$ \\
\hline \multirow[t]{5}{*}{$\overline{2013}$} & I & $0.0^{\mathrm{b}}$ & $0.0^{\mathrm{b}}$ & $0.0^{\mathrm{b}}$ & $0.0^{\mathrm{b}}$ & $0.0^{\mathrm{b}}$ & $0.0^{\mathrm{B}}$ & $5.6^{\mathrm{ef}}$ & $7.7^{\text {cd }}$ & $5.3^{\text {efg }}$ & $2.9^{\mathrm{ijk}}$ & $1.4^{\mathrm{jk}}$ & $4.6^{\mathrm{B}}$ \\
\hline & II & $0.0^{\mathrm{b}}$ & $0.0^{\mathrm{b}}$ & $0.0^{\mathrm{b}}$ & $0.0^{\mathrm{b}}$ & $0.0^{\mathrm{b}}$ & $0.0^{\mathrm{B}}$ & $6.0^{\mathrm{def}}$ & $5.0^{\mathrm{e}-\mathrm{h}}$ & $6.8^{\text {cde }}$ & $7.5^{\mathrm{cd}}$ & $0.0^{\mathrm{k}}$ & $5.1^{\mathrm{AB}}$ \\
\hline & III & $0.0^{\mathrm{b}}$ & $0.0^{\mathrm{b}}$ & $0.0^{\mathrm{b}}$ & $0.0^{\mathrm{b}}$ & $0.0^{\mathrm{b}}$ & $0.0^{\mathrm{B}}$ & $8.2^{\mathrm{c}}$ & $5.3^{\mathrm{efg}}$ & $1.0^{\mathrm{k}}$ & $14.1^{\mathrm{b}}$ & $0.0^{\mathrm{k}}$ & $5.7^{\mathrm{A}}$ \\
\hline & IV & $2.3^{\mathrm{a}}$ & $0.0^{\mathrm{b}}$ & $0.0^{\mathrm{b}}$ & $0.0^{\mathrm{b}}$ & $0.0^{\mathrm{b}}$ & $0.5^{\mathrm{A}}$ & $6.7^{\mathrm{def}}$ & $0.0^{\mathrm{k}}$ & $0.0^{\mathrm{k}}$ & $6.1^{\mathrm{def}}$ & $0.0^{\mathrm{k}}$ & $2.6^{\mathrm{C}}$ \\
\hline & $\mathrm{V}$ & $2.2^{\mathrm{a}}$ & $0.0^{\mathrm{b}}$ & $0.0^{\mathrm{b}}$ & $0.0^{\mathrm{b}}$ & $0.0^{\mathrm{b}}$ & $0.4^{\mathrm{A}}$ & $4.4^{\mathrm{f}-\mathrm{i}}$ & $0.0^{\mathrm{k}}$ & $5.6^{\mathrm{ef}}$ & $17.3^{\mathrm{a}}$ & $0.0^{\mathrm{k}}$ & $5.5^{\mathrm{A}}$ \\
\hline \multirow[t]{5}{*}{2014} & I & $0.4^{\mathrm{b}}$ & $1.9^{\mathrm{a}}$ & $0.0^{\mathrm{b}}$ & $0.0^{\mathrm{b}}$ & $0.0^{\mathrm{b}}$ & $0.5^{\mathrm{A}}$ & $0.6^{\mathrm{k}}$ & $3.1^{\mathrm{h}-\mathrm{k}}$ & $0.4^{\mathrm{k}}$ & $0.0^{\mathrm{k}}$ & $0.0^{\mathrm{k}}$ & $0.8^{\mathrm{DE}}$ \\
\hline & II & $0.0^{\mathrm{b}}$ & $0.4^{\mathrm{b}}$ & $0.0^{\mathrm{b}}$ & $0.0^{\mathrm{b}}$ & $0.0^{\mathrm{b}}$ & $0.1^{\mathrm{B}}$ & $0.4^{\mathrm{k}}$ & $3.2^{\text {hij }}$ & $0.0^{\mathrm{k}}$ & $0.0^{\mathrm{k}}$ & $0.0^{\mathrm{k}}$ & $0.7^{\mathrm{DE}}$ \\
\hline & III & $0.0^{\mathrm{b}}$ & $2.1^{\mathrm{a}}$ & $0.0^{\mathrm{b}}$ & $0.0^{\mathrm{b}}$ & $0.0^{\mathrm{b}}$ & $0.4^{\mathrm{A}}$ & $0.9^{\mathrm{k}}$ & $4.9^{\mathrm{e}-\mathrm{h}}$ & $0.0^{\mathrm{k}}$ & $0.0^{\mathrm{k}}$ & $0.0^{\mathrm{k}}$ & $1.2^{\mathrm{D}}$ \\
\hline & IV & $0.0^{\mathrm{b}}$ & $0.4^{\mathrm{b}}$ & $0.0^{\mathrm{b}}$ & $0.0^{\mathrm{b}}$ & $0.0^{\mathrm{b}}$ & $0.1^{\mathrm{B}}$ & $0.8^{\mathrm{k}}$ & $3.6^{\mathrm{ghi}}$ & $1.4^{\mathrm{jk}}$ & $0.0^{\mathrm{k}}$ & $0.0^{\mathrm{k}}$ & $1.2^{\mathrm{D}}$ \\
\hline & $\mathrm{V}$ & $0.0^{\mathrm{b}}$ & $0.7^{\mathrm{b}}$ & $0.0^{\mathrm{b}}$ & $0.3^{\mathrm{b}}$ & $0.0^{\mathrm{b}}$ & $0.2^{\mathrm{AB}}$ & $3.6^{\mathrm{ghi}}$ & $3.6^{\mathrm{ghi}}$ & $0.0^{\mathrm{k}}$ & $0.0^{\mathrm{k}}$ & $0.0^{\mathrm{k}}$ & $1.4^{\mathrm{D}}$ \\
\hline \multirow[t]{5}{*}{2015} & I & $0.0^{\mathrm{b}}$ & $0.0^{\mathrm{b}}$ & $0.0^{\mathrm{b}}$ & $0.0^{\mathrm{b}}$ & $0.0^{\mathrm{b}}$ & $0.0^{\mathrm{B}}$ & $0.4^{\mathrm{k}}$ & $0.3^{\mathrm{k}}$ & $0.0^{\mathrm{k}}$ & $0.0^{\mathrm{k}}$ & $0.0^{\mathrm{k}}$ & $0.1^{\mathrm{E}}$ \\
\hline & II & $0.0^{\mathrm{b}}$ & $0.0^{\mathrm{b}}$ & $0.0^{\mathrm{b}}$ & $0.0^{\mathrm{b}}$ & $0.0^{\mathrm{b}}$ & $0.0^{\mathrm{B}}$ & $0.0^{\mathrm{k}}$ & $0.3^{\mathrm{k}}$ & $0.0^{\mathrm{k}}$ & $0.0^{\mathrm{k}}$ & $0.0^{\mathrm{k}}$ & $0.1^{\mathrm{E}}$ \\
\hline & III & $0.0^{\mathrm{b}}$ & $0.0^{\mathrm{b}}$ & $0.0^{\mathrm{b}}$ & $0.0^{\mathrm{b}}$ & $0.0^{\mathrm{b}}$ & $0.0^{\mathrm{B}}$ & $0.4^{\mathrm{k}}$ & $0.0^{\mathrm{k}}$ & $0.0^{\mathrm{k}}$ & $0.0^{\mathrm{k}}$ & $0.0^{\mathrm{k}}$ & $0.1^{\mathrm{E}}$ \\
\hline & IV & $0.0^{\mathrm{b}}$ & $0.0^{\mathrm{b}}$ & $0.0^{\mathrm{b}}$ & $0.0^{\mathrm{b}}$ & $0.0^{\mathrm{b}}$ & $0.0^{\mathrm{B}}$ & $1.1^{\mathrm{k}}$ & $0.0^{\mathrm{k}}$ & $0.0^{\mathrm{k}}$ & $0.0^{\mathrm{k}}$ & $0.0^{\mathrm{k}}$ & $0.2^{\mathrm{E}}$ \\
\hline & $\mathrm{V}$ & $0.0^{\mathrm{b}}$ & $0.0^{\mathrm{b}}$ & $0.0^{\mathrm{b}}$ & $0.0^{\mathrm{b}}$ & $0.0^{\mathrm{b}}$ & $0.0^{\mathrm{B}}$ & $0.0^{\mathrm{k}}$ & $0.4^{\mathrm{k}}$ & $0.5^{\mathrm{k}}$ & $0.0^{\mathrm{k}}$ & $0.0^{\mathrm{k}}$ & $0.2^{\mathrm{E}}$ \\
\hline \multicolumn{14}{|l|}{ Mean } \\
\hline \multicolumn{2}{|l|}{2013} & $0.9^{\mathrm{A}}$ & $0.0^{\mathrm{B}}$ & $0.0^{\mathrm{B}}$ & $0.0^{\mathrm{B}}$ & $0.0^{\mathrm{B}}$ & $0.2^{\mathrm{A}}$ & $6.2^{\mathrm{B}}$ & $3.6^{\mathrm{C}}$ & $3.7^{\mathrm{C}}$ & $9.6^{\mathrm{A}}$ & $0.3^{\mathrm{E}}$ & $4.7^{\mathrm{A}}$ \\
\hline \multicolumn{2}{|l|}{2014} & $0.1^{\mathrm{B}}$ & $1.1^{\mathrm{A}}$ & $0.0^{\mathrm{B}}$ & $0.1^{\mathrm{B}}$ & $0.0^{\mathrm{B}}$ & $0.2^{\mathrm{A}}$ & $1.3^{\mathrm{D}}$ & $3.7^{\mathrm{C}}$ & $0.4^{\mathrm{E}}$ & $0.0^{\mathrm{E}}$ & $0.0^{\mathrm{E}}$ & $1.1^{\mathrm{B}}$ \\
\hline \multicolumn{2}{|l|}{2015} & $0.0^{\mathrm{B}}$ & $0.0^{\mathrm{B}}$ & $0.0^{\mathrm{B}}$ & $0.0^{\mathrm{B}}$ & $0.0^{\mathrm{B}}$ & $0.0^{\mathrm{B}}$ & $0.4^{\mathrm{E}}$ & $0.2^{\mathrm{E}}$ & $0.1^{\mathrm{E}}$ & $0.0^{\mathrm{E}}$ & $0.0^{\mathrm{E}}$ & $0.1^{\mathrm{C}}$ \\
\hline \multicolumn{14}{|c|}{ Mean for 2013-2015 } \\
\hline \multicolumn{2}{|l|}{ I } & $0.1^{\mathrm{B}}$ & $0.6^{\mathrm{A}}$ & $0.0^{\mathrm{B}}$ & $0.0^{\mathrm{B}}$ & $0.0^{\mathrm{B}}$ & $0.2^{\mathrm{A}}$ & $2.2^{\mathrm{EFG}}$ & $3.7^{\mathrm{C}}$ & $1.9^{\mathrm{FGH}}$ & $1.0^{\mathrm{HIJ}}$ & $0.5^{\mathrm{IJ}}$ & $1.9^{\mathrm{B}}$ \\
\hline \multicolumn{2}{|l|}{ II } & $0.0^{\mathrm{B}}$ & $0.1^{\mathrm{B}}$ & $0.0^{\mathrm{B}}$ & $0.0^{\mathrm{B}}$ & $0.0^{\mathrm{B}}$ & $0.0^{\mathrm{A}}$ & $2.1^{\mathrm{EFG}}$ & $2.8^{\mathrm{C}-\mathrm{F}}$ & $2.3^{\mathrm{EFG}}$ & $2.5^{\mathrm{DEF}}$ & $0.0^{\mathrm{J}}$ & $2.0^{\mathrm{AB}}$ \\
\hline \multicolumn{2}{|l|}{ III } & $0.0^{\mathrm{B}}$ & $0.7^{\mathrm{A}}$ & $0.0^{\mathrm{B}}$ & $0.0^{\mathrm{B}}$ & $0.0^{\mathrm{B}}$ & $0.1^{\mathrm{A}}$ & $3.2^{\mathrm{CDE}}$ & $3.4^{\mathrm{CD}}$ & $0.3^{\mathrm{IJ}}$ & $4.7^{\mathrm{B}}$ & $0.0^{\mathrm{J}}$ & $2.3^{\mathrm{A}}$ \\
\hline \multicolumn{2}{|l|}{ IV } & $0.8^{\mathrm{A}}$ & $0.1^{\mathrm{B}}$ & $0.0^{\mathrm{B}}$ & $0.0^{\mathrm{B}}$ & $0.0^{\mathrm{B}}$ & $0.2^{\mathrm{A}}$ & $2.9^{\mathrm{C}-\mathrm{F}}$ & $1.2^{\mathrm{GHI}}$ & $0.5^{\mathrm{IJ}}$ & $2.0^{\mathrm{EFG}}$ & $0.0^{\mathrm{J}}$ & $1.3^{\mathrm{C}}$ \\
\hline \multicolumn{2}{|l|}{$\mathrm{V}$} & $0.7^{\mathrm{A}}$ & $0.2^{\mathrm{B}}$ & $0.0^{\mathrm{B}}$ & $0.1^{\mathrm{B}}$ & $0.0^{\mathrm{B}}$ & $0.2^{\mathrm{A}}$ & $2.7^{\mathrm{C}-\mathrm{F}}$ & $1.3^{\mathrm{GHI}}$ & $2.0^{\mathrm{EFG}}$ & $5.8^{\mathrm{A}}$ & $0.0^{\mathrm{J}}$ & $2.4^{\mathrm{A}}$ \\
\hline \multicolumn{2}{|l|}{$\mathrm{x}$} & $0.3^{\mathrm{A}}$ & $0.4^{\mathrm{A}}$ & $0.0^{\mathrm{B}}$ & $0.0^{\mathrm{B}}$ & $0.0^{\mathrm{B}}$ & - & $2.6^{\mathrm{B}}$ & $2.5^{\mathrm{B}}$ & $1.4 \mathrm{C}$ & $3.2^{\mathrm{A}}$ & $0.1^{\mathrm{D}}$ & - \\
\hline
\end{tabular}

Values denoted by the same letters for years do not differ significantly at $5 \%$ error (Duncan's test)

I control, II Asahi SL, III Bio-Algeen S-90, IV Kelpak SL, V Trifender WP, Blaue Blaue St. Galler, HB HB Red

two years of research) and the resulting low soil moisture content did not support the spread of soft rot (symptoms of the disease were observed on individual tubers). According to Kurzawińska and Mazur (2010), potato tubers infected with Phytophthora infestans are more susceptible to diseases caused by $P$. carotovorum subsp. carotovorum and fungi of the genus Fusarium. In this study, the prevalence of dry rot was also relatively low. The percentage of infected tubers was highest in cv. Irga, after harvest (13\% on a mass basis) in the Kelpak SL treatment and after 5 months of storage (around 28\%) in the Trifender WP treatment (Table 3). An analysis of the average values of the infection index across cultivars and years revealed that potatoes cv. Irga were most affected by dry rot (only $5 \%$ on a mass basis) after harvest in 2013. In the remaining cultivars treated with biostimulants, the infection index did not exceed $1 \%$. The above products limited the spread of dry rot in tubers cv. Satina, whereas Bio-Algeen S-90, Kelpak SL and Trifender WP exerted a stimulating influence on potatoes cv. Irga. The disease progressed during storage, and its symptoms were most visible in potatoes harvested in the first growing season, which could be attributed to high precipitation between late June and the second half of July. The severity of the disease differed between the analyzed cultivars in the first and last year of the study, and HB Red (and Valfi in the last year of the experiment) was least affected. Dry rot symptoms were reduced in potatoes cvs. Irga and Blaue St. Geller harvested from plants treated with Bio-Algeen S-90 and Kelpak SL biostimulants, respectively. An analysis of mean values across treatments revealed higher severity of dry rot at harvest in the Kelpak SL treatment and after storage in the Trifender WP treatment. In the work of Patel and Saraf (2017), Trichoderma asperellum MSST effectively protected tomatoes against Fusarium oxysporum f. sp. lycopersici. According to Surekha et al. (2014), the above can be attributed to the induction of phenolic compounds by the Trichoderma viride fungus in Vigna mungo plants infected with $F$. oxysporum and Alternaria alternata fungal pathogens. In a study by Reiter et al. (2012), maleic hydrazide (MH-30) had a minor 
Table 5 Fungi isolated from potato tubers after harvest and 5-month storage (\%)

\begin{tabular}{|c|c|c|c|c|c|c|}
\hline \multirow[t]{2}{*}{ Fungi } & & \multicolumn{5}{|c|}{ After harvest } \\
\hline & & I & II & III & IV & $\mathrm{V}$ \\
\hline \multirow[t]{6}{*}{ Pathogens } & Alternaria spp. & 6.86 & 8.23 & 8.79 & 5.50 & 12.15 \\
\hline & Colletotrichum coccodes & 0.81 & 1.85 & 2.38 & 0.80 & 0.85 \\
\hline & Fusarium spp., Gibberella spp. & 12.90 & 9.67 & 12.16 & 10.30 & 10.45 \\
\hline & Helminthosporium solani & 0 & 0 & 0 & 0 & 0.21 \\
\hline & Rhizoctonia solani & 6.86 & 5.76 & 4.77 & 4.80 & 4.47 \\
\hline & Pythium spp. & 4.24 & 1.44 & 0 & 5.00 & 1.71 \\
\hline \multicolumn{2}{|c|}{ Antagonists (Gliocladium spp., Trichoderma spp., Paecilomyces spp.) } & 6.86 & 10.91 & 7.48 & 10.50 & 7.68 \\
\hline \multicolumn{2}{|c|}{$\begin{array}{l}\text { Mucorales (Mortierella spp., Mucor spp., Rhizopus spp., Zygorhynchus } \\
\text { spp.) }\end{array}$} & 3.24 & 6.79 & 3.48 & 4.00 & 3.84 \\
\hline \multicolumn{2}{|c|}{ Penicillium spp. } & 49.62 & 45.68 & 50.98 & $\begin{array}{l}49.7 \\
0\end{array}$ & 46.06 \\
\hline \multicolumn{2}{|l|}{ Others } & 8.61 & 9.67 & 9.96 & 9.40 & 12.58 \\
\hline \multicolumn{2}{|c|}{ Total [number of isolates] } & 496 & 486 & 461 & 500 & 469 \\
\hline \multicolumn{7}{|c|}{ After storage } \\
\hline \multirow[t]{5}{*}{ Pathogens } & Alternaria spp. & 5.18 & 4.52 & 3.62 & 3.22 & 5.36 \\
\hline & Colletotrichum coccodes & 1.64 & 2.86 & 1.33 & 0.64 & 2.14 \\
\hline & Fusarium spp., Gibberella spp. & 2.63 & 6.36 & 1.90 & 3.65 & 6.07 \\
\hline & Rhizoctonia solani & 0 & 0.82 & 0.96 & 1.72 & 0.36 \\
\hline & Pythium spp. & 0.36 & 0.41 & 0.19 & 0.64 & 0.18 \\
\hline \multicolumn{2}{|c|}{ Antagonists (Gliocladium spp., Trichoderma spp., Paecilomyces spp.) } & 6.27 & 7.77 & 3.05 & 3.65 & 5.54 \\
\hline \multicolumn{2}{|c|}{$\begin{array}{l}\text { Mucorales (Mortierella spp., Mucor spp., Rhizopus spp., Zygorhynchus } \\
\text { spp.) }\end{array}$} & 1.82 & 1.85 & 4.57 & 3.65 & 2.14 \\
\hline \multicolumn{2}{|c|}{ Penicillium spp. } & 79.29 & 70.07 & 81.33 & 78.11 & 75.35 \\
\hline \multicolumn{2}{|l|}{ Others } & 2.81 & 5.34 & 3.05 & 4.72 & 2.86 \\
\hline \multicolumn{2}{|c|}{ Total [numer of isolates] } & 549 & 487 & 525 & 466 & 560 \\
\hline
\end{tabular}

Others: after harvest (Absidia sp., Acremonium strictum, Aspergillus flavus, A. niger, Aureobasidium pullulans, Botrytis cinerea, Chaetomium funicola, C. spirale, Cylindrocarpon destructans, Endothia sp., Epiccocum sp., Gliomastix fimbriatum, G. murorum, Monilia sp., Periconia sp., Sporotrichioides olivaceum, Sporormia sp., Yeast-like fungi, Non-sporulating cultures); after storage (Chaetomium funicola, C. globosum, Epiccocum sp., Periconia sp., Phompa sp., Sporotrichioides olivaceum, Yeast-like fungi, Non-sporulating cultures)

I control, II Asahi SL, III Bio-Algeen S-90, IV Kelpak SL, V Trifender WP, Blaue Blaue St. Galler, HB HB Red

protective effect against tuber rot. The extracts of Stokeyia indica and Padina pavonia brown algae and Solieria robusta red alga were effective in counteracting Fusarium solani infections in Abelmoschus esculentus (Sultana et al. 2005) and Glycine max (Sultana et al. 2011). Root infections caused by $F$. solani in Solanum melongena L. were effectively mitigated by extracts of Spatoglossum variabile, Polycladia indica and Melanothamnus afaqhusainii (Baloch et al. 2013) and powdered extracts of Padina gymnospora, Sargassum latifolium and Hydroclathrus clathratus applied to soil (Ibraheem et al. 2017).

Late blight symptoms were observed sporadically in only $1 \%$ of the mass of potatoes cv. Irga at harvest in 2013 and potatoes cv. Satina in 2014. The remaining cultivars were not affected by the disease (Table 4). Disease symptoms were observed in stored tubers harvested in every year of the study, excluding Blaue St. Galler and HB Red in the last two years of the experiment. The above could be indicative of a latent infection with Phytophthora infestans.
Potatoes cv. Blaue St. Galler were most significantly affected by late blight ( $10 \%$ on a mass basis) in 2013, probably due to high precipitation levels in June and July, which were twice higher than those noted in the corresponding months of 2014 and 2015 (similarly to dry rot). Kelpak SL and Trifender WP biostimulants reduced the severity of late blight in stored tubers cv. Satina. Potatoes cv. HB Red harvested from plants treated with biostimulants were not affected by the disease. The severity of late blight increased in tubers cv. Blaue St. Galler harvested from all treatments. An analysis of the average values of the infection index in the experimental treatments demonstrated that Bio-Algeen S-90 and Trifender WP exacerbated disease symptoms in stored potatoes. In a previous study, Bio-Algeen S-90 and Kelpak SL growth regulators and the Asahi SL biostimulant reduced the severity of late blight and dry rot in potato tubers (Cwalina-Ambroziak et al. 2015). 


\section{Fungi Isolated from Potato Tubers}

The analyzed pathogens were more abundant on potato tubers at harvest (from 26.4 to $31.7 \%$ in the Kelpak SL treatment and the control treatment, respectively) than after 5 months of storage (from 8 to $15 \%$ in Bio-Algeen S-90 and Asahi SL treatments, respectively) (Table 5). The predominant pathogens were Fusarium spp. and Gibberella spp. fungi as well as Alternaria spp. which colonized $12 \%$ of tubers harvested from plants not treated with biostimulants and from plants treated with the Trifender WP biostimulant. Rhizoctonia solani, Colletotrichum coccodes and Pythium spp. were less frequently isolated, and the presence of Helminthosporium solani was noted sporadically (only at harvest). In Poland, dry rot is most often caused by F. sulphureum, Gibberella pulicaris and Haematonectria hematococca, and mechanically damaged potatoes are more susceptible to infection (Kurzawińska and Gajda 2002). The above pathogens are components of fungal communities colonizing potato tubers, and $C$. coccodes was the predominant species in a previous study by Cwalina-Ambroziak et al. (2015), unlike in the current experiment. Pathogens of the genera Fusarium and Gibberella accounted for only $6 \%$ of all fungi isolated from stored potatoes (Asahi SL treatment), whereas pathogens of the genus Alternaria had a 5\% share of the fungal community in the control treatment and the Trifender WP treatment. The applied growth regulators limited the occurrence frequency of the causative agents of dry rot and black scurf after harvest. CwalinaAmbroziak et al. (2015) isolated the smallest number of pathogens from potatoes harvested from plants treated with Asahi SL. In the present study, a higher number of antagonists were isolated from potatoes grown from plants treated with biostimulants at harvest than after 5 months of storage. The tested biostimulants also increased the populations of saprophytes of the order Mucorales after harvest and after 5 months of storage. Their share of fungal isolates did not exceed 7\% (Asahi SL treatment) in freshly harvested potatoes and 5\% (Bio-Algeen S-90 treatment) in stored tubers. The large community of Penicillium species continued to increase during storage, and their abundance was not influenced by the applied biostimulants.

\section{The Content of Chlorogenic Acids}

Total chlorogenic acid was present in higher concentrations in the skin than in the flesh of potato tubers analyzed at harvest and after storage. At harvest, the chlorogenic acid content of potato skin ranged from $77.9 \mathrm{mg} \mathrm{g}^{-1} \mathrm{DM}$ (Irga, Kelpak SL) to $458.7 \mathrm{mg} \mathrm{g}^{-1} \mathrm{DM}$ (Valfi, Trifender WP) in 2013, and the chlorogenic acid content of potato flesh ranged from $8.0 \mathrm{mg} \mathrm{g}^{-1} \mathrm{DM}$ (Irga, Bio-Algeen S-90) to $121.2 \mathrm{mg} \mathrm{g}^{-1} \mathrm{DM}$ (Blaue St. Galler, Asahi SL) in 2014 (Table 6). Our results are consistent with the findings of other authors (Albishi et al. 2013; Ezekiel et al. 2013). In the present study, the concentrations of chlorogenic acid (mean values for cultivars) in the skin and flesh of potato tubers were similar across years. Significantly higher concentrations of chlorogenic acid were found in potato cultivars with blue-purple- and red-colored flesh than in those with yellow- and cream-colored flesh. Hamouz et al. (2013) noted higher levels of chlorogenic acid in potato tubers in warm locations with regular periods of drought than in high-altitude locations. Nemś et al. (2015) observed the highest concentrations of phenolic compounds in potato cultivars with purple flesh (Blaue Cango and Blaue Annelise), 2-fold lower in potato cultivars with red-colored flesh (Herbie 26 and Rote Emma), and 3- and 5-fold lower in traditional potato cultivars (Vineta and Fresco).

In a study by Zarzecka et al. (2019), the phenolic content of potato tubers ranged from 150.1 to $166.5 \mathrm{mg} \mathrm{kg}^{-1}$ fresh weight, and it was significantly affected by cultivar and the applied herbicides and biostimulants (Asahi SL and Kelpak SL). In the current experiment, a significant increase in chlorogenic acid content, relative to control, was noted at harvest in all years the study in the skin of potato tubers $\mathrm{cv}$. Valfi, Blaue St. Galler and HB Red treated with Asahi SL and Trifender WP, and in the last two years in cv. Satina treated with Trifender WP. The chlorogenic acid content of potato flesh increased significantly in all years of the study in cv. Valfi, Blaue St. Galler and HB Red treated with Asahi SL (except for cv. Valfi in 2013), in cv. Valfi and Blaue St. Galler treated with Trifender WP, and in the first and last year of the study in cv. Satina and HB Red treated with Trifender WP, respectively.

After storage, a minor decrease in the chlorogenic acid content of potato skin was noted in cv. Valfi in the first two years of the study (treatment with Asahi SL) and in cv. Satina in 2014 (treatment with Trifender WP). A greater decrease in the concentrations of chlorogenic acid was observed in potato flesh in cv. Irga in 2013 (treatment with Bio-Algeen S-90), in cv. Satina in 2015 (treatment with Asahi SL), in cv. Valfi and Blaue St. Galler-32.6\% in all years of the study (treatment with Asahi SL), and in cv. Valfi-27.2\% in 2013 (treatments with Bio-Algeen S-90 and Trifender WP) (Table 7). Madiwale et al. (2011) reported an increase in the concentrations of chlorogenic acid, caffeic acid and sinapinic acid in potato tubers stored for 90 days at a temperature of $3^{\circ} \mathrm{C}$. Galani et al. (2017) demonstrated that the levels of total phenolic compounds increased and then decreased in potato tubers stored at room temperature $\left(25-32^{\circ} \mathrm{C}\right)$. The cited authors observed fluctuations in the content of phenolic compounds throughout storage at 4 and $15^{\circ} \mathrm{C}$, which was higher on the last day of observation than on the first day. Contrary results were reported by Singh and Saldana (2011) who noted losses in 


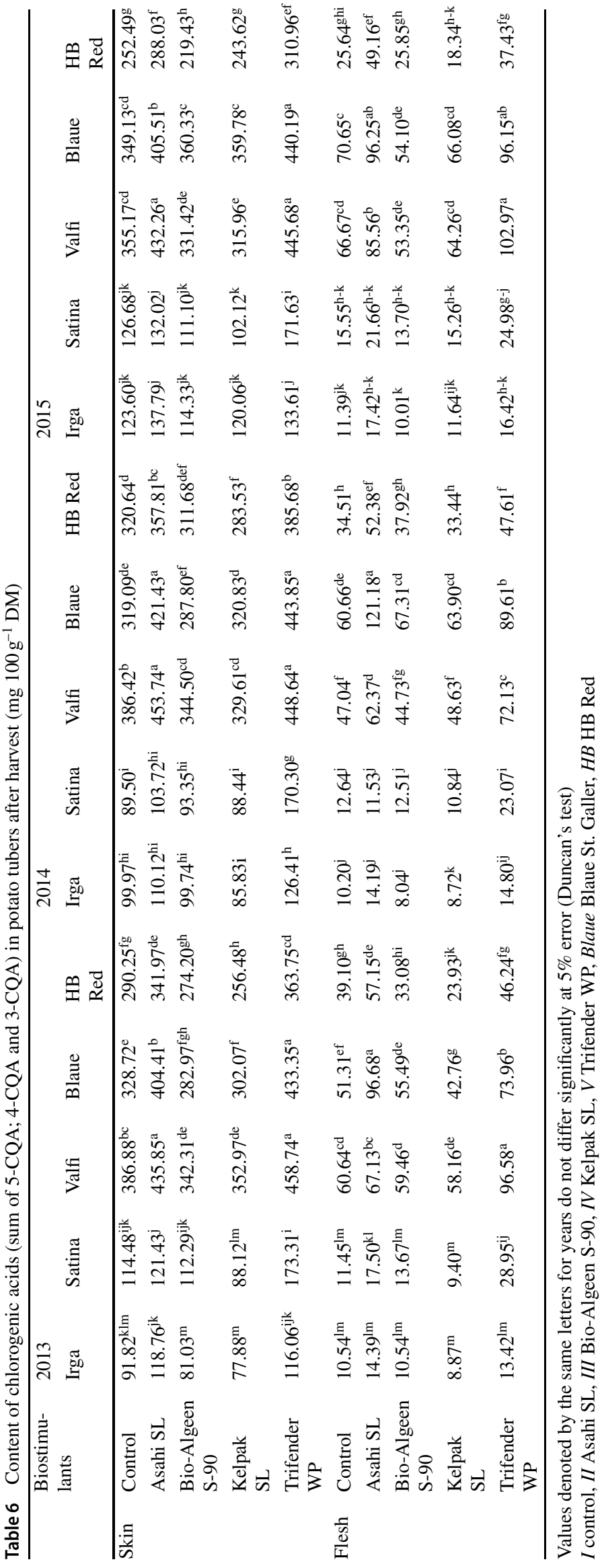




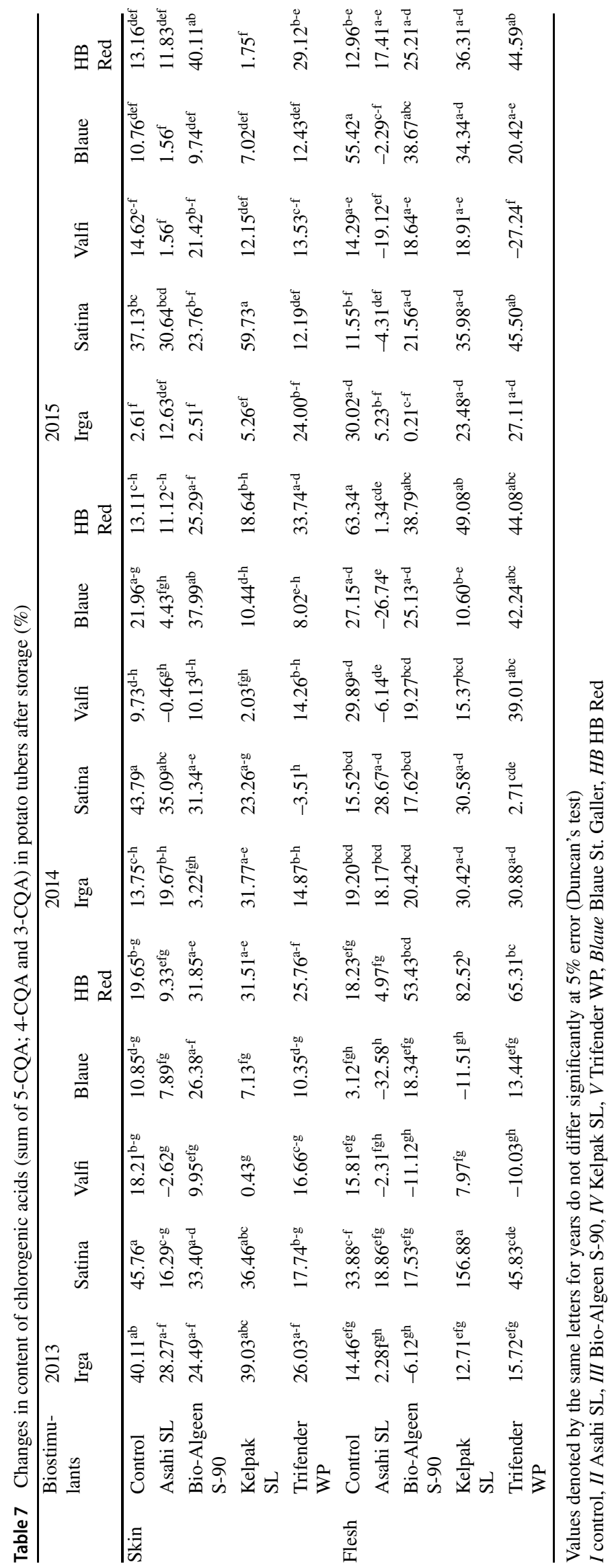




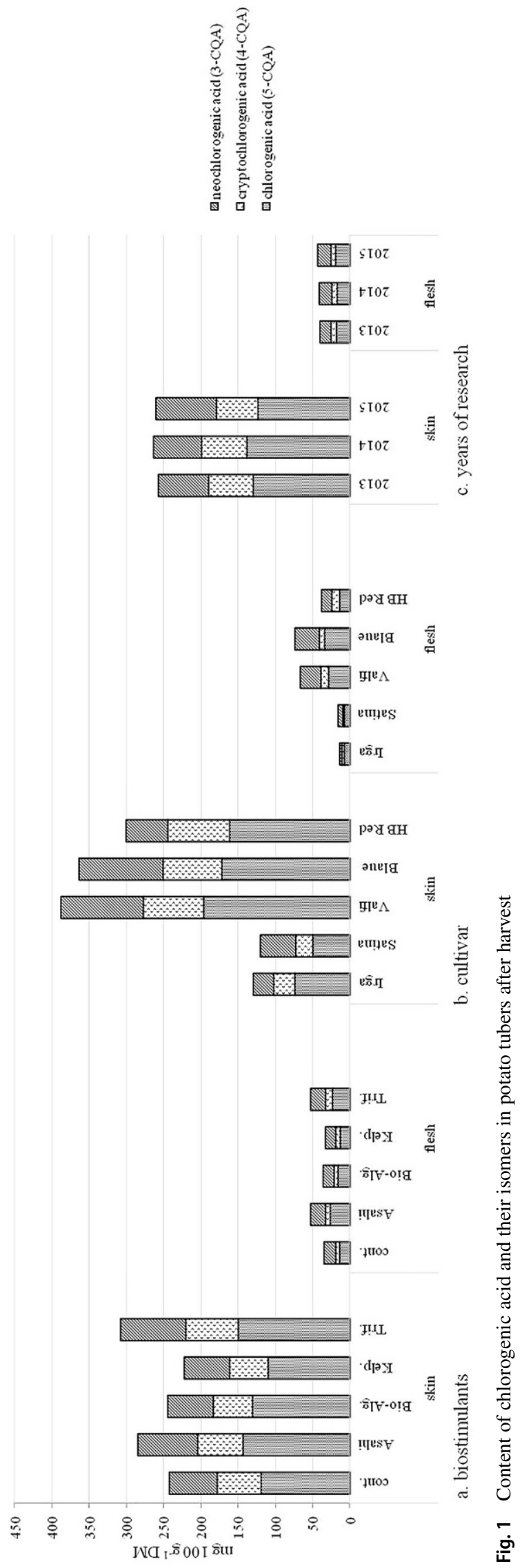




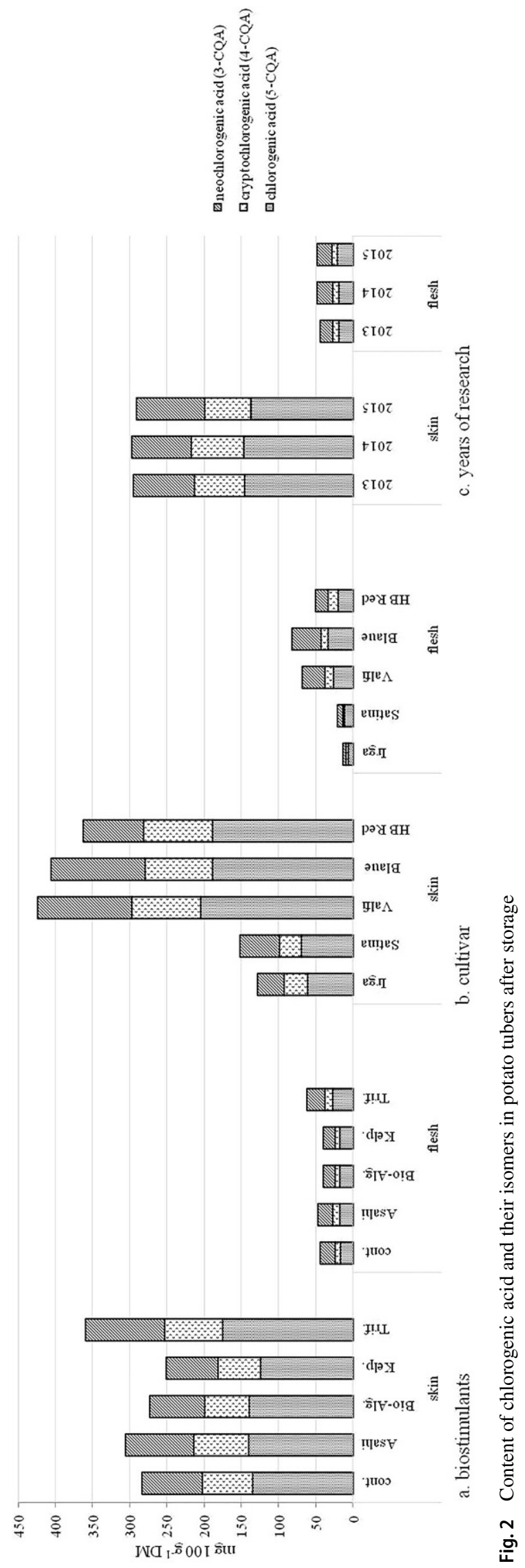


phenolic compounds during storage, especially at high temperatures (around $10^{\circ} \mathrm{C}$ ) (Andre et al. 2009). In the present experiment, the experimental factors-cultivars, biostimulants and years of the study modified the content of individual chlorogenic acids in the skin of potato tubers analyzed at harvest and after storage (the concentrations of chlorogenic acids in potato flesh were low which resulted in small differences) (Fig. 1 and 2). The predominant phenolic acid was chlorogenic acid (5-caffeoylquinic acid/5-CQA). The content of neochlorogenic acid (3-caffeoylquinic acid/3CQA), in comparison with cryptochlorogenic acid (4-caffeoylquinic acid /4-CQA), was somewhat higher in the skin of potato tubers than in the flesh in all years of the study, it was higher in treatments with Asahi SL and Trifender WP, and in cv. Valfi and Blaue St. Galler. According to Maldonado et al. (2014), chlorogenic acid accounts for $90 \%$ of all phenolic compounds in potato skin, where it is present in the form of three major isomers (mentioned above).

\section{Conclusions}

The severity of potato diseases was significantly influenced by weather and genetic factors conditioning resistance in the analyzed cultivars, whereas the applied biostimulants exerted minor effects. The harvested and stored potatoes were most affected by common scab, in particular in the second growing season. Black scurf, dry rot and late blight did not pose a significant threat, and soft rot symptoms were observed sporadically. A minor increase in the severity of the analyzed diseases was noted during storage. In harvested and stored potatoes, tubers cvs. Irga and HB red were least infected with Streptomyces spp. and Dickeya spp., and tubers cvs. Valfi, Blaue St. Galler and HB Red were least infected with Rhizoctonia solani. After 5 months of storage, tubers cvs. Valfi and HB Red were least infected with Phytophthora infestans, and tubers cvs. Valfi, HB Red and Blaue St. Galler-with Fusarium spp. The applied treatments decreased the severity of dry rot in freshly harvested potatoes cv. Satina. In stored potatoes, common scab symptoms were minimized in tubers cv. Satina in the Asahi SL treatment, the severity of late blight was decreased in potatoes cv. Satina in Kelpak SL and Trifender WP treatments and in potato cv. Valfi in the Bio-Algeen S-90 and Kelpak SL treatments, the severity of dry rot was limited in potatoes $\mathrm{cv}$. Irga in the Bio-Algeen S-90 treatment and in potatoes cv. Blaue St. Galler in the Kelpak SL treatment, and black scurf symptoms were mitigated in tubers cv. Irga in Asahi SL and Kelpak SL treatments. A higher number of pathogens were isolated from freshly harvested than from stored potatoes. Fusarium spp. and Gibberella spp. predominated, whereas Alternaria spp. and Rhizoctonia solani were less frequently isolated. The applied treatments decreased the occurrence frequency of the causative agents of dry rot and black scurf after harvest, and stimulated the development of antagonists of fungal pathogens. Total chlorogenic acid, with a predominance of 5-caffeoylquinic acid (5-CQA), was present in higher concentrations in the skin than in the flesh of potato tubers at harvest and after storage. Higher concentrations of chlorogenic acids were found in potato tubers $\mathrm{cv}$. Valfi, Blaue St. Galler and HB Red (with blue-purple- and red-colored flesh) than in cv. Irga and Satina (with yellowand cream-colored flesh). The chlorogenic acid content of potato tubers increased in response to the application of the Asahi SL biostimulant and Trifender WP.

Conflict of interest M. Głosek-Sobieraj, B. Cwalina-Ambroziak, A. Waśkiewicz, K. Hamouz and A. Perczak declare that they have no competing interests.

Open Access This article is distributed under the terms of the Creative Commons Attribution 4.0 International License (http:// creativecommons.org/licenses/by/4.0/), which permits unrestricted use, distribution, and reproduction in any medium, provided you give appropriate credit to the original author(s) and the source, provide a link to the Creative Commons license, and indicate if changes were made.

\section{References}

Albishi T, John J, Al-Khalifa A, Shahidi F (2013) Phenolic content and antioxidant activities of selected potato varieties and their processing by-products. J Funct Foods 5:590-600. https://doi.org/10. 1016/j.jff.2012.11.019

Amado I, Franco D, Sánchez M, Zapata C, Vázquez J (2014) Optimisation of antioxidant extraction from Solanum tuberosum potato peel waste by surface response methodology. Food Chem 165:290-299. https://doi.org/10.1016/j.foodchem.2014.05.103

Andre C, Schafleitner R, Guignard C, Oufir M, Aliaga C, Nomberto G, Hoffmann L, Hausman J, Evers D, Larondelle Y (2009) Modification of the health-promoting value of potato tubers field grown under drought stress: emphasis on dietary antioxidant and glycoalkaloid contents in five native andean cultivars (Solanum tuberosum L.). J Agric Food Chem 57:599-609. https://doi.org/10.1021/ jf8025452

Anees M, Tronsmo A, Edel-Hermann V, Gautheron N, Faloya V, Steinberg C (2010) Biotic changes in relation to local decrease in soil conduciveness to disease caused by Rhizoctonia solani. Eur J Plant Pathol 126:29-41. https://doi.org/10.1007/s10658-0099517-0

Bains PS, Bennypaul HS, Lynch DR, Kawchuk LM, Schaupmeyer CA (2002) Rhizoctonia disease of potatoes (Rhizoctonia solani): fungicidal efficacy and cultivar susceptibility. Am J Potato Res 79:99-106. https://doi.org/10.1007/BF02881518

Baloch GN, Tariq S, Ehteshamul-Haque S, Athar M, Sultana V, Ara J (2013) Management of root diseases of eggplant and watermelon with the application of asafoetida and seaweeds. J Appl Bot Food Qual 86:138-142. https://doi.org/10.5073/JABFQ.2013.086.019

Bellumori M, Innocenti M, Michelozzi M, Cerretani L, Mulinacci N (2017) Coloured-fleshed potatoes after boiling: promising sources of known antioxidant compounds. J Food Compos Anal 59:1-7. https://doi.org/10.1016/j.freeradbiomed.2003.09.022

Buysens C, César V, Ferrais F, de Boulois HD, Declerck S (2016) Inoculation of Medicago sativa cover crop with Rhizophagus irregularis and Trichoderma harzianum increases the yield of subse- 
quently-grown potato under low nutrient conditions. Appl Soil Ecol 105:137-143. https://doi.org/10.1016/j.apsoil.2016.04.011

Calvo P, Nelson L, Kloeppeer JW (2014) Agricultural uses of plant biostimulants. Plant Soil 383:3-41. https://doi.org/10.1007/ s11104-014-2131-8

Chojnacka K, Saeid A, Michalak I (2012) The possibilities of the application of algal biomass in the agriculture. Chemik 66(11):1235-1248.

Cullen DW, Lees AK (2007) Detection of the nec1 virulence gene and its correlation with pathogenicity in Streptomyces species on potato tubers and in soil using conventional and real time PCR. J Appl Microbiol 102(4):1082-1094. https://doi.org/10.1111/j. 1365-2672.2006.03146.x

Cwalina-Ambroziak B, Głosek-Sobieraj M, Kowalska E (2015) The effect of plant growth regulators on the incidence and severity of potato diseases. Pol J Nat Sci 30(1):5-20.

Esserti S, Smaili A, Rifai LA, Koussa T, Makroum K, Belfaiza M, Kabil EM, Faize L, Burgos L, Alburquerque N, Faize M (2017) Protective effect of three brown seaweed extracts against fungal and bacterial diseases of tomato. J Appl Phycol 29(2):1081-1093. https://doi.org/10.1007/s10811-016-0996-z

Ezekiel R, Singh N, Sharma S, Kaur A (2013) Beneficial phytochemicals in potato-a review. Food Res Int 50:487-496. https://doi. org/10.1016/j.foodres.2011.04.025

Gachango E, Hanson LE, Rojas A, Hao JJ, Kirk WW (2012) Fusarium spp. causing dry rot of seedpotato tubers in Michigan and their sensitivity to fungicides. Plant Dis 96:1767-1774. https://doi.org/ 10.1094/PDIS-11-11-0932-RE

Galani JHY, Mankad PM, Shah AK, Patel NJ, Acharya RR, Talati JG (2017) Effect of storage temperature on vitamin C, total phenolics, UPLC phenolic acid profile and antioxidant capacity of eleven potato (Solanum tuberosum) varieties. Hortic Plant J 3(2):73-89. https://doi.org/10.1016/j.hpj.2017.07.004

Gardan L, Gouy C, Christen R, Samson R (2003) Elevation of three subspecies of Pectobacterium carotovorum to species level: Pectobacterium atrosepticum sp. nov., Pectobacterium betavasculorum sp. nov. and Pectobacterium wasabiae sp. nov. Int J Syst Evol Microbiol 53(2):381-391. https://doi.org/10.1099/ijs.0.02423-0

Gliszczyńska-Świgło A, Ciska E, Pawlak-Lemańska K, Chmielewski J, Borkowski T, Tyrakowska B (2006) Changes in the content of health-promoting compounds and antioxidant activity of broccoli after domestic processing. Food Addit Contam 23(11):1088-1098. https://doi.org/10.1080/02652030600887594

Grosch R, Scherwinski K, Lottmann J, Berg G (2006) Fungal antagonists of the plant pathogen Rhizoctonia solani: selection, control efficacy and influence on the indigenous microbial community. Mycol Res 110:1464-1474. https://doi.org/10.1016/j.mycres. 2006.09.014

Głosek-Sobieraj M, Cwalina-Ambroziak B, Hamouz K (2018) The effect of growth regulators and a biostimulator on the health status, yield and yield components of potatoes (Solanum tuberosum L.). Gesunde Pflanzen 70(1):1-11. https://doi.org/10.1007/s10343017-0407-7

Hamouz K, Lachman J, Hejtmánková K, Pazderů K, Čížek M, Dvořák $P$ (2010) Effect of natural and growing conditions on the contentof phenolics in potatoes with different flesh colour. Plant Soil Environ 56(8):368-374.

Hamouz K, Lachman J, Pazderu K, Hejtmankova K, Cimr J, Musilova J, Pivec V, Orsak M, Svobodova A (2013) Effect of cultivar, location and method of cultivation on the content of chlorogenic acid in potatoes with different flesh colour. Plant Soil Environ 59(10):465-471.

Horoszkiewicz-Janka J, Michalski T (2006) The effect of protective treatments on plumpness of grain, germinating capacity and specific composition of fungi isolated from grain of barley and oat. Prog Plant Prot 46(1):417-423. (in Polish)
Horoszkiewicz-Janka J, Jajor E (2006) The effect of seed dressing on healthiness of barley, wheat and rape in early development stages. J Res Appl Agric Eng 51(2):47-53. (in Polish)

Ibraheem BMI, Hamed SM, Abd Elrhman AA, Farag FM, AbdelRaouf N (2017) Antimicrobial activities of some brown macroalgae against some soil borne plant pathogens and in vivo management of Solanum melongena root diseases. Aust J Basic Appl Sci 11:157-168.

Jaulneau V, Lafitte C, Corio-Costet MF, Stadnik MJ, Salamagne S, Briand X, Esquerré-Tugayé MT, Dumas B (2011) An Ulva armoricana extract protects plants against three powdery mildew pathogens. Eur J Plant Pathol 131:393-401. https://doi.org/10. 1007/s10658-011-9816-0

Kosanić M, Ranković B, Stanojković T (2015) Biological activities of two macroalgae from Adriatic coast of Montenegro. Saudi J Biol Sci 22(4):390-397. https://doi.org/10.1016/j.sjbs.2014.11.004

Kumar CS, Raju D, Sarada VL, Rengasamy R (2008) Seaweed extracts control the leaf spot disease of the medicinal plant Gymnema sylvestre. Indian J Sci Technol 1:93-94.

Kurzawińska H, Gajda I (2002) Fungi settling dry rotting potato tubers. Biul IHAR 223/224:315-319. (in Polish)

Kurzawińska H, Mazur S (2010) Biotechnical preparations applied during vegetation period and the occurrence of fungal diseases on potato tuber peel. Prog Plant Prot 50(4):2039-2043. (in Polish)

Larkin RP, Honeycutt CW (2006) Effects of different 3-year cropping systems on soil microbial communities and Rhizoctonia diseases of potato. Phytopathology 96(1):68-79. https://doi.org/10.1094/ PHYTO-96-0068

Leiminger J, Frank M, Wenk C, Poschenrieder G, Kellermann A, Schwarzfischer A (2013) Distribution and characterization of Streptomyces species causing potato common scab in Germany. Plant Pathol 62(3):611-623. https://doi.org/10.1111/j.1365-3059. 2012.02659.x

Lenc L (2006) Effect of pre-sprouting of potato tubers on occurrence of Rhizoctonia solani Kühn on sprouts and tubers of six organically grown potato cultivars. J Res Appl Agric Eng 51(2):104-107. (in Polish)

Lutomirska B (2008) The influence of meteorological factors on tuber infection with common scab. Prog Plant Prot 48(1):216-220. (in Polish)

Madiwale GP, Reddivari L, Holm DG, Vanamala J (2011) Storage elevates phenolic content and antioxidant activity but suppresses antiproliferative and pro-apoptotic properties of colored-flesh potatoes against human colon cancer cell lines. J Agric Food Chem 59:8155-8166. https://doi.org/10.1021/jf201073g

Maldonado AFS, Mudge E, Gänzle M, Schieber A (2014) Extraction and fractionation of phenolic acids and glycoalkaloids from potato peels using acidified water/ethanol-based solvents. Food Res Int 65:27-34. https://doi.org/10.1016/j.foodres.2014.06.018

Nemś A, Miedzianka J, Pęksa A, Kita A (2015) Prohealthy compounds content in potatoes varieties of different flesh colour. Bromatol Chem Toksykol XLVIII(3):473-478. (in Polish)

Patel S, Saraf M (2017) Biocontrol efficacy of Trichoderma asperellum MSST against tomato wilting by Fusarium oxysporum f. sp. lycopersici. Arch Phytopathol Plant Prot 50:228-238. https://doi. org/10.1080/03235408.2017.1287236

Paulert R, Ebbinghaus D, Urlass C, Moerschbacher M (2010) Priming of the oxidative burst in rice and wheat cell cultures by ulvan, a polysaccharide from green macroalgae, and enhanced resistance against powdery mildew in wheat and barley plants. Plant Pathol 59:634-642. https://doi.org/10.1111/j.1365-3059.2010.02300.x

Paulert R, Talamini V, Cassolato JEF, Duarte MER, Noseda MD, Smania A, Stadnik MJ (2009) Effects of sulphated polysaccharide and alcoholic extracts from green seaweeds Ulva fasciata on anthracnose severity and growth of common bean (Phaseolus vulgaris L.). J Plant Dis Prot 116:263-270. https://doi.org/10.1007/ BF03356321 
Raj TS, Graff KH, Suji HA (2016) Bio chemical characterization of a brown seaweed algae and its efficacy on control of rice sheath blight caused by Rhizoctonia solani Kühn. Int J Trop Agric 34:429-439.

Reiter MS, Rideout SL, Freeman JH (2012) Nitrogen fertilizer and growth regulator impacts on tuber deformity, rot, and yield for Russet potatoes. Int J Agron 1-7. https://doi.org/10.1155/2012/ 348754

Sawicka B (1999) The infuence of synthetic growth regulators Mival and Moddus in potato cultivation on tuber infection with Streptomyces sp. and Rhizoctonia solani. Prog Plant Prot 39(2):616-620. (in Polish)

Singh P, Saldaña M (2011) Subcritical water extraction of phenolic compounds from potato peel. Food Res Int 44:2452-2458. https:// doi.org/10.1016/j.foodres.2011.02.006

Sultana V, Baloch GN, Ara J, Ehteshamul-Haque S, Tariq RM, Athar M (2011) Seaweeds as an alternative to chemical pesticides for the management of root diseases of sunflower and tomato. J Appl Bot Food Qual 84:162-168.

Sultana V, Ehteshamul-Haque S, Ara J, Athar M (2005) Comparative efficacy of brown, green and red seaweeds in the control of root infecting fungi and okra. Int J Environ Sci Technol (Tehran) 2:129-132. https://doi.org/10.1007/BF03325866

Surekha CH, Neelapu NRR, Prasad BS, Sankar GP (2014) Induction of defense enzymes and phenolic content by Trichoderma viride in Vigna mungo infested with Fusarium oxysporum and Alternaria alternata. Int J Agric Sci Res 4:31-40.

Sławiak M, Łojkowska E, Van Der Wolf JM (2009) First report of bacterial soft rot on potato caused by Dickeya sp. (syn. Erwinia chrysanthemi) in Poland. Plant Pathol 58(4):794. https://doi.org/ 10.1111/j.1365-3059.2009.02028.x

Tambascio C, Covacevich F, Lobato MC, de Lasa C, Caldiz DO, Dosio GAA, Andreu AB (2014) The application of K phosphites to seed tubers enhanced emergence, early growth and mycorrhizal colonization in potato (Solanum tuberosum). Am J Plant Sci 5:132-137. https://doi.org/10.4236/ajps.2014.51017

Terry LA, Joyce DC, Adikaram NKB, Kambay PBS (2014) Preformed antifungal compounds in strawberry fruit and flower tissues. Postharvest Biol Technol 31:201-210. https://doi.org/10.1016/j. postharvbio.2003.08.003

Thornton MK, Lee J, John R, Olsen NL, Navarre DA (2013) Influence of growth regulators on plant growth, yield, and skin color of specialty potatoes. Am J Potato Res 90(3):271-283. https://doi.org/ $10.1007 / \mathrm{s} 12230-013-9302-7$

Tierno R, Ruiz de Galarreta JI (2016) Breeding for nutritional quality and pest resistance: potential of a set of non-commercial tetraploid potato cultivars with purple and red flesh. Rev Latinoam Papa 20(1):9-17.

Waterer D (2010) Influence of growth regulators on skin colour and scab diseases of red-skinned potatoes. Can J Plant Sci 90(5):745-753. https://doi.org/10.4141/CJPS10055

Wierzbowska J, Cwalina-Ambroziak B, Głosek M, Sienkiewicz S (2015) Effect of biostimulators on yield and selected chemical properties of potato tubers. J Elementol 20(3):757-768. https:// doi.org/10.5601/jelem.2014.19.4.799

Youssef SA, Tartoura KA, Abdelraouf GA (2016) Evaluation of Trichoderma harzianum and Serratia proteamaculans effect on disease suppression, stimulation of ROS-scavenging enzymes and improving tomato growth infected by Rhizoctonia solani. Biol Control 100:79-86. https://doi.org/10.1016/j.biocontrol.2016.06. 001

Zarzecka K, Gugała M, Sikorska A, Mystkowska I, Baranowska A, Niewęgłowski M, Dołęga H (2019) The effect of herbicides and biostimulants on polyphenol content of potato (Solanum tuberosum L.) tubers and leaves. J Saudi Soc Agric Sci 18:102-106. https://doi.org/10.1016/j.jssas.2017.02.004

Łacicowa B (1970) Investigations on Helminthosporium sorokinianum (=H. sativum $)$ strains and on the resistance of spring barley varieties to this pathogenic factor. Acta Mycol 6(2):184-248. (in Polish)

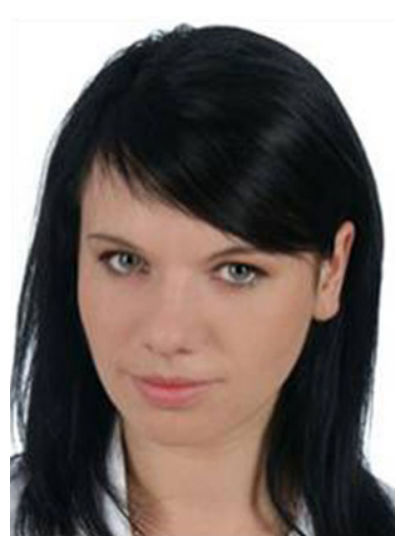

Małgorzata Głosek-Sobieraj was born on 10.07.1987 in Ostrołęka (Poland). She is a $\mathrm{PhD}$ student at the Department of Entomology, Phytopathology and Molecular Diagnostics at the University of Warmia and Mazury in Olsztyn (research supervisor is professor Bożena Cwalina-Ambroziak). Her research field is the health status of plants, including the potato; biological control against pathogens; a mechanism of disease resistance in plants. 